\title{
A Survey of Algorithmic and Hardware Optimization Techniques for Vision Convolutional Neural Networks on FPGAs
}

\author{
Arish Sateesan • Sharad Sinha - Smitha \\ K.G. • A. P. Vinod
}

\begin{abstract}
In today's world, the applications of convolutional neural networks $(\mathrm{CNN})$ are limitless and are employed in numerous fields. The CNNs get wider and deeper to achieve near-human accuracy. Implementing such networks on resource constrained hardware is a cumbersome task. CNNs need to be optimized both on hardware and algorithmic levels to compress and fit into resource limited devices. This survey aims to investigate different optimization techniques of Vision CNNs, both on algorithmic and hardware level, which would help in efficient hardware implementation, especially for FPGAs.
\end{abstract}

Keywords FPGA · Hardware Optimization · Convolutional Neural Networks, Resource Constrained Hardware

This work is supported by the MOE Singapore Tier-1 grant under Grant No.: 2017-T1-001-192 (RG28/17). The first author did most of the work while at NTU Singapore.

Arish Sateesan

imec-COSIC/ES\&S, ESAT

KU Leuven, 3000 Leuven, Belgium

E-mail: arish.sateesan@kuleuven.be

Sharad Sinha

Indian Institute of Technology Goa

Farmagudi, Ponda, India-403401

E-mail: sharad@iitgoa.ac.in

Smitha K.G.

Nanyang Technological University

50 Nanyang Ave, Singapore-639798

E-mail: smitha@ntu.edu.sg

\section{A. P. Vinod}

Indian Institute of Technology Palakkad

Ahalia Integrated Campus

Palakkad, Kerala, India-678557

E-mail: vinod@iitpkd.ac.in 


\section{Introduction}

Convolutional Neural Networks (CNNs) are fast becoming a key instrument in computer vision applications, which can provide near-human accuracy. The quest for accuracy makes the network models extremely deep with millions of parameters and this increases the storage capacity requirements and makes them computationally intensive. As a result of this, to implement these in hardware, it is very much important to choose a suitable hardware platform. Central Processing Units (CPU) alone cannot handle such computations, hence a hardware accelerator is absolutely necessary to reduce the load of the CPUs.

The obvious choice of a hardware accelerator for CNNs is Graphics Processing Units (GPU) because of its very high computational capability. Though GPU implementations of neural networks (NNs) deliver the best throughput, currently more interest is on moving CNNs into Field Programmable Gate Arrays (FPGA), for example the Catapult project [1] and Project Brainwave from Microsoft [2], because of the rapid improvement in FPGA technology. Despite the popularity of GPUs in deep learning implementations, FPGAs are emerging as an alternative mainly because of advantages like high performance per watt, parallelism, and the flexibility in model level optimizations compared to the the fixed architectures of GPUs. In low-power environments, FPGAs are more efficient than the power draining GPUs, and hence suitable for mobile devices and resource limited platforms. FPGAs are more flexible for CNNs as they can support any custom modifications in the algorithms, for example, the custom low-precision datatypes which will be difficult for GPUs to handle. Also, FPGAs can be used as a standalone system to implement any design, especially with the advances of System-on-Chip (SoC) FPGAs.

Despite of these advantages of FPGAs, deployment of CNNs into FPGAs is quite tiresome because of the unavailability of a suitable high-level framework specifically for FPGAs. Translation of CNNs to FPGAs often require low-level/RTL programming along with precise resource allocation, which can be a quite lengthy process and unavailability of an efficient model-to-FPGA compiler coule be a limiting factor. In the case of CPUs/GPUs, a number of high-level frameworks are available for compiling the algorithms into hardware design. FPGAs and other embedded hardware platforms also suffer from lack of sufficient computational resources to handle the high computational requirements of CNNs efficiently. There has been numerous studies in the field of hardware optimization for resource-constrained devices, but these works lag behind in comparison with the rapid advances in algorithmic development. Hence, it is very much important to have optimizations in the structural and algorithmic levels too to make the algorithms suitable for embedded/resource-constrained hardware. Hardware/software co-design approaches are a better solution for such problems and many such promising works are published recently. In this paper, we conduct a thorough study about different optimization techniques and hardware/software co-design approaches to target the implementation of vision based CNNs on resource-limited devices like FPGAs. Even though it is difficult to choose and include all the work related to this area, we have tried our best to include the works which are having a great impact.

This paper is organized in eight sections as shown in figure 1 . Section 2 discusses the state-of-the-art networks in vision-based CNNs. Section 3 


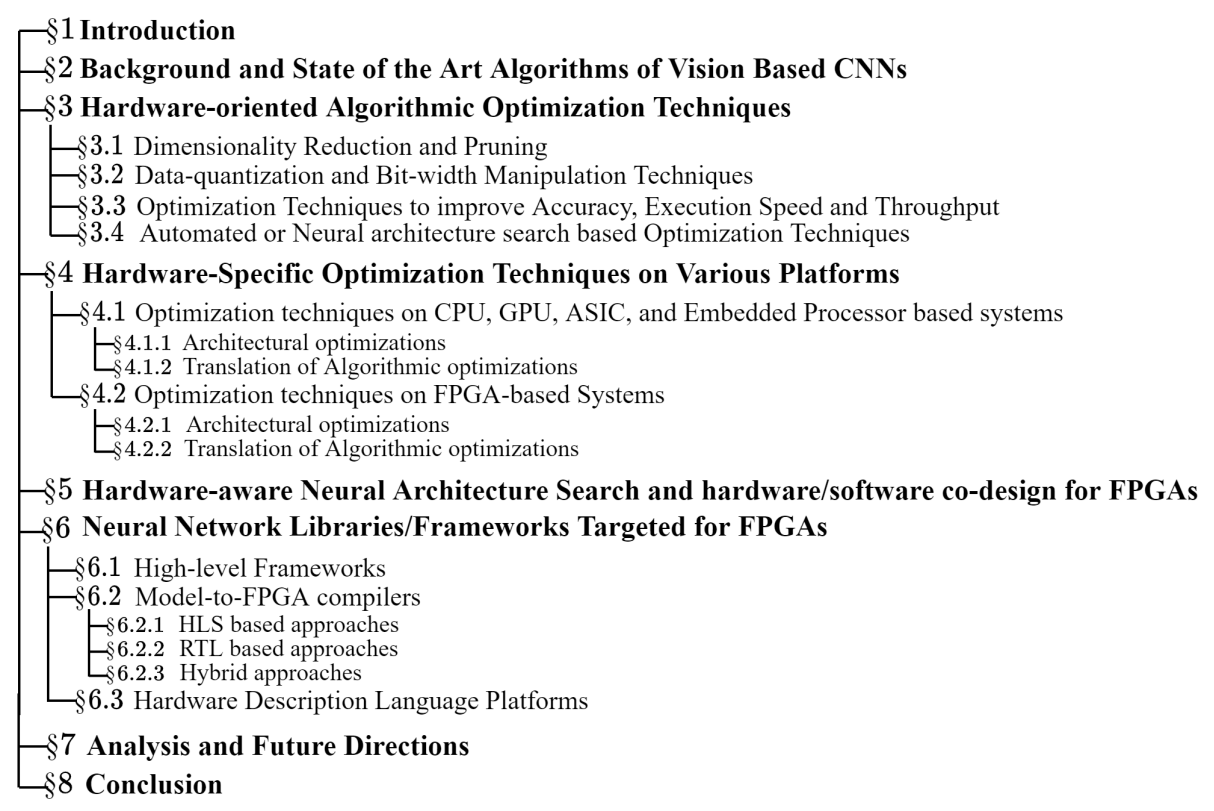

Fig. 1 Organization of the paper

and section 4 presents a detailed study of hardware-oriented algorithmic and hardware-specific optimization techniques respectively. A discussion on hardware-aware neural architecture search and co-design approaches is presented in section 5. Section 6 presents the libraries and frameworks targeting FPGA specifically. Discussion about the study and future trends in hardware implementation of CNNs is given in section 7 . Section 8 concludes the paper.

\section{Background and State of the Art Algorithms of Vision Based CNNs}

In 1958, Dr. Frank Rosenblatt came up with 'The embryo' [3]-the computer which was able to differentiate between left and right after 50 trials- which lead to Perceptron, a probabilistic model for information storage and organization in the brain. McCulloch and Pitts introduced the first artificial neuron to the world in 1943 [4]. Numerous research works on machine learning and NNs were carried out and many models were introduced later to mimic the human brain. However, a revolution in neural network research happened when Geoffrey Hinton proposed the backpropagation algorithm in 1985 [5]. Yann LeCun, in 1998 introduced LeNet-5 [6], which used the backpropagation algorithm to perform document recognition. Till then there were few researches available about the application of CNNs in computer vision. LeNet provided a new direction to the research in NNs. The classification accuracy is quite high for LeNet considering the fact that it has $60 \mathrm{~K}$ parameters and used sigmoid and tanh functions - which are computationally intensive compared to current modern choices like ReLU (Rectified Linear Unit). 
AlexNet by Krizhevsky [7] achieved a Top-1 and Top-5 error rates of $37.5 \%$ and $16.4 \%$ respectively on ImageNet dataset and was the best among the rest at that time. It has 60 million parameters and $650 \mathrm{~K}$ neurons. It used multiple GPUs for convolution operations in 5 convolution layers. AlexNet has three fully connected (FC) layers and a regularization method called dropout is used to avoid overfitting. Overlapped pooling is used to reduce error rates. Network in Network (NIN) architecture by Min Lin [8] made the network even deeper. NIN introduced inception modules, which drastically reduced the feature map size. NIN paved the path for new approaches of designing convolutional filters. One of the most efficient and most praised network models inspired by the NIN architecture is GoogLeNet $[9]$.

VGG network (Visual Geometry Group) [10] came up with an improvement in the depth of the network by making the network deeper with the addition of more convolution layers. The area of the network is balanced in these layers by using $3 \times 3$ filters in all layers. VGGNet has 139 million parameters, which is not high despite being deep when comparing with any relatively shallow network with large filter widths. However, GoogLeNet has a slight edge over VGG in terms of accuracy and number of parameters, because GoogLeNet [9] reduces the number of layers in the network using inception modules and hence the number of parameters. Residual Networks (ResNet) [11] has 8 times as many layers as VGG, but has less complexity. ResNet was built upon the basic model of AlexNet and VGG by using residual model with batch normalization followed by each convolution layer. The residual model introduced the skip or shortcut connections in layers. In simple networks, the error rate increases with increase in the number of layers. However, ResNet provides improved error rates with increasing number of layers. GoogLeNet and VGG showed Top-5 error rates of 7.89 and 8.43 respectively in 2014 ImageNet[12] competition, while ResNet-152 with a depth of 152 layers showed an error rate of 4.49 .

Following the footsteps of GoogleNet, models like SqueezeNet[13], YOLO [14] and MobileNets [15] made it possible to reduce the model size to less than $1 \mathrm{MB}$, which can be fit into a small hardware platform. The backbone of MobileNets also is a modified version of the inception module. Facebook Artificial Intelligence research introduced DenseNet [16] with very fewer parameters and computations shows better results compared to ResNets. ShuffleNet [17] also outperform most efficient models like mobileNets and SqueezeNet in terms of accuracy. mobileNet-V3 [18], MobileNetV2 [19] and ShuffleNetV2 [20] can be considered as candidates for the most efficient and state-of-the-art models at the moment. More detailed explanation of NIN, GoogLeNet, SqueezeNet, MobileNets, ShuffleNet and DenseNet models in terms of architectural features are given in section 3 .

The automated approach of neural network design, Neural Architecture Search (NAS), introduced by Google brings some of the most efficient networks. These automated networks like NASNet [21], PNasNet [22], MnasNet [23], AmoebaNet [24], and Darts [25] shows that these models can perform better than some of the most efficient models like MobileNetV2 and ShuffleNetV2. These networks are described in detail in sections 3. YOLO [14] is the state-of-the-art real-time object detection model and it can achieve a speed of 45 Frames Per Second (FPS), and its tiny version FAST YOLO can achieve a speed of 155 FPS. A modified GoogLeNet is the core of YOLO. It takes object detection as 
a regression problem and uses only a single feedforward network. Fast YOLO is the fastest detection model in PASCAL VOC detection and it is twice as fast as any other real-time detector as of now.

A simple comparison in terms of accuracy, input size and model size of some of the state-of-the-art models are given in table 1. All models except LeNet are evaluated using ImageNet dataset and LeNet is evaluated using MNIST dataset[6]. From table 1, we can see that GoogleNet stands out in terms of model size and top-5 accuracy. ResNet also has much improved accuracy and has only about 3x size compared to GoogleNet. This is the reason why most of the recent researches follow these two network models.

Table 1 Performance and Model Parameter Comparison for Some Popular Neural Networks

\begin{tabular}{|l|c|c|c|c|c|c|}
\hline Model & Input Size & Weights & MAC & $\begin{array}{c}\text { Top-1 } \\
\text { Accuracy }\end{array}$ & $\begin{array}{c}\text { Top-5 } \\
\text { accuracy }\end{array}$ & $\begin{array}{c}\text { Model } \\
\text { Size }\end{array}$ \\
\hline [6] LeNet (MNIST) & $28 \times 28$ & $60 \mathrm{~K}$ & $2.3 \mathrm{M}$ & NA & NA & $1.72 \mathrm{MB}$ \\
\hline [7] AlexNet (ImageNet) & $227 \times 227$ & $61 \mathrm{M}$ & $724 \mathrm{M}$ & $57.2 \%$ & $80.3 \%$ & $240 \mathrm{MB}$ \\
\hline$[10]$ VGG-16 (ImageNet) & $224 \times 224$ & $138 \mathrm{M}$ & $15.3 \mathrm{G}$ & $71.5 \%$ & $91.2 \%$ & $528 \mathrm{MB}$ \\
\hline$[9]$ GoogleNet (ImageNet) & $224 \times 224$ & $6.8 \mathrm{M}$ & $1.55 \mathrm{G}$ & $69.8 \%$ & $89.3 \%$ & $26 \mathrm{MB}$ \\
\hline$[11]$ ResNet-50 (ImageNet) & $224 \times 224$ & $25.5 \mathrm{M}$ & $3.9 \mathrm{G}$ & $75.2 \%$ & $93 \%$ & $87 \mathrm{MB}$ \\
\hline$[9]$ ResNet-152 (ImageNet) & $224 \times 224$ & $60 \mathrm{M}$ & $11.3 \mathrm{G}$ & $77.6 \%$ & $93.8 \%$ & $220 \mathrm{MB}$ \\
\hline$[26]$ Inception V3 & $224 \times 224$ & $23.6 \mathrm{M}$ & $5.7 \mathrm{G}$ & $78.2 \%$ & $94.1 \%$ & NA \\
\hline$[27]$ Xception & $224 \times 224$ & $22.8 \mathrm{M}$ & $5.7 \mathrm{G}$ & $79.0 \%$ & $94.5 \%$ & NA \\
\hline
\end{tabular}

For implementing CNNs on hardware, specialized hardware architectures are necessary to meet requirements of power consumption, execution time, memory footprint, resource utilization, re-usability, and scalability. Many different approaches have been put forward to tackle the inefficiency of hardware accelerator designs in terms of precision, resources, memory, power etc. It is important to mention that the optimization methods are not only necessary on the hardware level, but also on the structural and algorithmic levels. Especially for the deployment on FPGAs, it is important to have an optimized design because of the unavailability of a suitable high-level framework which can convert complex algorithms into efficient hardware designs. However, compilation of complex algorithms to hardware is difficult. A hardware/software co-design approach would be much efficient while considering the deployment of neural networks. Hence, approaches like hardware-aware NAS [28] are the need of the hour.

The range of optimization on hardware is always limited, unless there are advances in FPGA process technologies. Starting with the roofline model approach [29], conventional approaches like loop-unrolling [30], pipelining and approaches like systolic array architectures [31-33] are commonly used techniques. Along with such techniques, combination of optimizations like data-quantization, acceleration interfacing optimization [34], data-path optimizations like data-reuse, efficient use of on-chip memory, etc. can help to improve the performance on hardware. Though hardware description languages provide better design flexibility for hardware designer, it is more efficient in terms of design time and complexity to have a high-level language like OpenCL which can provide optimized libraries for compiling algorithms onto hardware as mentioned in $[35,36]$. The advances in model-to-FPGA frameworks like DeepBurning [37] is the most preferred design requirement for the present times. Our main motive for this survey is to study the 
optimization techniques in terms of both algorithmic and implementation levels, which could be helpful in analyzing an efficient hardware accelerator design for FPGAs.

\section{Hardware-oriented Algorithmic Optimization Techniques}

As mentioned in the previous section, it is very much important to have an optimized design not only in the implementation level, but also in the algorithmic and structural aspects of the network. The algorithmic-optimization techniques cover the structural and algorithmic level approaches in CNNs which are hardware-friendly. Since this paper focuses only on implementations of trained models, the algorithmic approaches to optimize training, like back-propagation and gradient descent calculations, are not covered in detail. Different techniques to reduce redundancy and sparsity, to improve the execution speed and throughput of CNN layers, and unsupervised and automated models are discussed in this section.

\subsection{Dimensionality Reduction and Pruning}

As the amount of data generated is growing exponentially, the problem of having too many variables/dimensions which are very similar and of little value can lead to serious performance issues like increased storage space, increased computational time, etc. Considering limited amount of fast memory and resources of FPGAs, simplification of CNNs are utmost important. There are mainly two methods to get the best result out of the data. One method is to reduce the dimensions or in other words data transformation from high-dimensional space to low-dimensional space, which is termed as Feature Projection or Dimensionality Reduction. Another method is by eliminating the insignificant variables without a significant loss in information, which is called Feature Selection. There are many methods like Principal Component Analysis (PCA), Auto Encoders, etc. to reduce the dimensions of a network. In this survey, we concentrate mainly on techniques related to Neural Networks and hardware optimization. Hence, a detailed explanation of feature selection techniques is not included, though some of the methods like regularization are mentioned.

One of the main problems of CNNs to deal with is the large number of parameters which requires a large memory footprint. Network Pruning [38-40] is a method to effectively reduce the network size. This approach concentrates on reducing the number of connections which are redundant to reduce the network complexity. Pruning is an iterative process and it is necessary to obtain the minimum number of connections. This same process applied to connections can be applied to neurons as well to remove redundant neurons after pruning connections. Pruning the FC layers reduces the size of the network drastically, while pruning in convolutional layers reduces the memory bandwidth [41]. The deeper the layer is, the more it will get pruned. The best way to prune a large network is to train the network first and then do the pruning [42]. Page et. al [40] introduces five pruning techniques to reduce the sparsity of CNNs. Pruning can be done at various levels like the number of layers, the number of connections, the number of neurons or the number of weights [43]. Pruning based on weight reduction was 
introduced in [44] based on Rumelhart's minimal networks idea. Tiled CNNs [45] is another parameter reduction method based on weights. Reagen et al [46] use pruning methods to remove insignificant neurons to reduce the power consumption.

One of the most impressive and effective ways of compression of neural network is Deep Compression [42,47], which introduces a 3-step pruning process. The first step involves learning the important connections by training a normal network. The second step involves removing all the connections which are having weights below a threshold level. The final step is retraining the sparse network obtained from the first two steps. Techniques like regularization and dropout are applied to increase the accuracy and prevent overfitting while retaining the network. This results in the reduction of the number of weights and computation by $12 \mathrm{x}$ and $12 \mathrm{x}, 12 \mathrm{x}$ and $6 \mathrm{x}, 9 \mathrm{x}$ and $3 \mathrm{x}$, and $12 \mathrm{x}$ and $5 \mathrm{x}$ respectively for LeNet-300-100, LeNet-5, AlexNet and VGG-16. The authors presented an improved version of deep compression [47] which uses pruning to reduce the network size $9 \mathrm{x}$ to $13 \mathrm{x}$ followed by weight quantization which makes reduction in size to $27 \mathrm{x}-31 \mathrm{x}$ which is then followed by Huffman coding which improves the overall reduction to $35 \mathrm{x}-49 \mathrm{x}$, all with no loss in accuracy. This helps to reduce the model size from 240MB to 6.9MB and store the model into On-chip memory.

Feature hashing [48] is an effective technique for dimensionality reduction. Hashing efficiently vectorizes features of high dimensional documents. Also, feature hashing effectively reduces the storage required. Chen et. al [48] proposes a NN architecture named HashedNets using hashing. Weight sharing is also employed in HashedNet [48] which uses hash function to calculate shared weight for each connection. For computer vision applications targeting embedded devices, differebt quantization techniques are employed in [49] to compress the network, especially dense connected layers. This work also analyses different vector quantization techniques like binarizing the parameters, scalar quantization using K-means clustering, and structured quantization using product quantization or residual quantization. The authors in the work [49] suggest that applying K-means clustering gives better results than matrix factorization methods as mentioned in [50], [51], and [52], and this followed by structured quantization can improve the results further. The evaluation is done using ZFNet model [53] for ImageNet dataset. Separate evaluation for different quantization methods and Singular Value Decomposition (SVD) are performed and the results show that using K-means clustering, 8-16x compression can be obtained with a loss in accuracy within $0.5 \%$. A further compression up to $24 \mathrm{x}$ can be obtained by K-means followed by structured quantization methods with an accuracy loss within $1 \%$.

In Inception network [9], the number of layers in a network and hence the computational cost are reduced by using NIN architecture [8]. NIN [8] replaced the generalized linear model of convolutional filters with nonlinear multi linear Perceptrons named MLPconv. i.e. instead of using a single filter, the MLPconv uses a neural network itself to produce a better level of abstraction for the latent features which a linear filter cannot provide. The output of the last MLPconv layer gives the activation maps equal to the number of classes to be predicted. This in turn makes the fully connected layers very much needless and can be replaced by average pooling which provides the raw scores of the classes and can be directly fed to softmax layer for prediction. The fully connected layers contains almost $90 \%$ of the parameters used in a network and by eliminating the fully connected networks the size of the network is reduced drastically. In inception network, the sizes of 
filters used in NIN architecture are reduced to $1 \times 1,3 \times 3$ and $5 \times 5$ and the number of layers can be reduced while using the same convolution, in its naive form. One problem with naive form is that the $3 \times 3$ and $5 \times 5$ convolution operations can be expensive with large number of filters. To eliminate this problem, dimension reduction is applied by employing $1 \mathrm{x} 1$ convolutions before applying the $3 \mathrm{x} 3$ and $5 \times 5$ filters. Using $1 \times 1$ filter before $5 \times 5$ filters reduces the number of parameters from 120 million to 12.4 million. Inception modules with dimension reduction gives very optimized design compared to naive form. Even though inception modules gives better optimization, it is beneficial to use inception modules only at higher layers for better memory efficiency.

Modified versions of Inception network from GoogLeNet - Inception-V2 [54], Inception-V3 [26], and Inception-V4 are proposed later. Inception-V3 is optimized for computer vision applications. A very efficient optimization technique is used to reduce the grid size by using two parallel strides of convolution and pooling, and then concatenates both in order to avoid the representational bottleneck. These optimizations and label smoothing performed individually forms the Inception-V2 and it is much more stable to variations than GoogLeNet and computation cost is only 2.5x higher than GoogLeNet, but still better than VGG. All these optimizations performed together forms the Inception-V3. Inception-V3 tops in both top-5 accuracy and top- 1 accuracy compared to other models and versions of GoogLeNet and VGGNet. Inception-V4 [54] is introduced after the introduction of Inception-ResNet-v1 and Inception-ResNet-v2 which combines the Residual Networks and the earlier versions of Inception Networks.

SqueezeNet [13] which is aimed for embedded applications makes the best use of inception module. The design is based on 3 strategies, where the first step replaces $3 \times 3$ filters with $1 \times 1$ filters and then decreases the number of input channels to $3 \times 3$ filters using squeeze layers. SqueezeNet claims of reducing the model size of AlexNet to less than $0.5 \mathrm{MB}$ so that it can be stored in the on-chip memory of FPGA devices. SqueezeNet, even with single precision floating point format, has a size of $4.8 \mathrm{MB}$, while SqueezeNet with data type 8 bit and 6 bit has a size of $0.66 \mathrm{MB}$ and $0.47 \mathrm{MB}$ respectively which is 363 and 510 times compressed compared to original size. The Top-1 accuracy is also higher than the original model and any of the compressed model, which is very impressive. SqueezeDet [55] also uses the SqueezeNet model with the addition of more layers followed by ConvDet convolution layer. SqueezeDet is targeted for real-time object detection and hence the accuracy is more important. With the addition of few more layers, accuracy of Squeezenet model is improved even though the size increases to under $8 \mathrm{MB}$.

MobileNets [15], MobileNetV2 [19], and MobileNetV3 [18] from Google are specifically aimed for mobile and embedded applications. MobileNets uses depthwise separable convolution [27] -a form of factorized convolutions, which can reduce the cost of the convolutional layer by a factor of $\mathrm{N}$, where $\mathrm{N}$ is the number of channels. In this model, the standard convolution operations are broken down to depthwise convolutions and pointwise or $1 \mathrm{x} 1$ convolutions. Depthwise separable convolutions make significant reduction in cost (reduce the multiply-adds by $8.55 \mathrm{x}$ and the parameters by $6.97 \mathrm{x}$ ) and the network is made even smaller by adding width multiplier and resolution multiplier parameters. Compared to other state of the art models, MobileNets is proved to be better for applications like geolocalization, fine grained recognition, face 
attribute classifier, object detection etc. MobileNetV2 [19] proposes a novel framework called SSDLite to provide enhanced performance when compared to MobileNetsV1. To implement semantic segmentation in mobile devices, a reduced version of DeepLabv3 [56] called mobile DeepLabv3 is introduced. MobileNetV2 is built upon MobileNets with the addition of linear bottleneck between layers and shortcut connections between bottlenecks using residual connections. The performance evaluation of MobileNetV2 for ImageNet on Google Pixel 1 phone shows a speedup of $1.5 \mathrm{x}$ and parameter reduction of $1.2 \mathrm{x}$ compared to MobileNetV1. In object detection, MobileNetV2+SSDLite shows $35 \%$ improvement in speed compared to mobilenetV1+SSDLite with less number of parameters. MobileNetV3[18] further improved the architecture for low-resource devices, which uses a combination of hardwareaware network architecture search (NAS) and the NetAdapt algorithm. MobileNetV3 introduced two models MobileNetV3-Large and MobileNetV3-Small which are targeted for high and low resource devices respectively. MobileNetV3-Large is $3.2 \%$ more accurate compared to MobileNetV2 while providing a $20 \%$ improvement in latency. Whereas MobileNetV3-Small is $6.6 \%$ more accurate compared to MobileNetV2 with almost similar latency.

ShuffleNet [17] is targeted for mobile devices with limited computing ability and it outperforms SqueezeNet [13] and YOLO [15] in terms of parameter size and accuracy. A shuffleNet unit is actually a residual block which consists of a pointwise 1x1 group convolution unit followed by a Channel shuffle unit, depthwise convolution unit and a final $1 \times 1$ pointwise group convolution unit. Channel shuffle unit reduces the degradation of accuracy and computational cost caused by pointwise convolutions. A $3 \times 3$ average-pooling is added to the shortcut path and elementwise addition is replaced by channel concatenation to enlarge channel dimension. ShuffleNetV2 [20] eliminated the limitations in shuffleNet [17] like increase in multiply-accumulate (MAC) operations in group convolutions and bottleneck structures by introducing a new idea of channel split. The input feature channels are split into 2 branches and one branch remains as identity and other branch contains two 1x1 non-group convolutions and a depthwise convolution. These branches are concatenated followed by channel shuffle. ShuffleNetV2 was designed to work under a computational budget of $40 \mathrm{M}$ FLOPs. ShuffleNetV2 outperforms some of the best models like MobileNet v2 [19], DenseNet [16], Xception [27] and CondenseNet [57] in terms of error \% in all the desirable computational budget in both ARM CPU and GPU. But, GPU speed in batches/sec is better for mobileNet V2.

A method to eliminate pooling and FC layers by replacing those with convolutional layers is proposed in the All Convolutional Net [58]. This model tried two approaches to replace pooling layer. One approach removes the pooling layer and increases the strides in the convolution layer preceded by it. The second approach replaces the pooling layer with a convolution layer having stride greater than one and number of output channels equal to number of input channels. The first approach downgraded the performance whereas the second approach shows promising results. The fully connected layers were replaced by simple $1 x 1$ convolutions and this helps to reduce the number of parameters.

DenseNet [16] introduced dense blocks and these dense blocks are stacked together to form a deep network, just like the inception modules and residual blocks in GoogleNet and ResNet. DenseNet closely follows ResNet and each layer 
is connected to every other layer in a feed forward manner. It prunces redundant connections between layers and one dense layer takes all preceding layers' outputs as inputs and the output is fed into subsequent dense layers. This in fact helps to eliminate the vanishing gradient problem, because of the reuse of features. Unlike ResNets, DenseNet does not perform summation to combine features, but concatenation is done instead. One advantage of such a dense network is that it requires fewer parameters. DenseNet has only 12 filters per layer and this makes the layers very narrow and this keeps the addition of feature maps to 'collective knowledge' very small and the remaining feature maps stay unchanged. Comparing with ImageNet, CIFAR[59]-10, CIFAR-100, and SVHN[60] datasets, DenseNets provide the best percentage error rates compared to other competing methods with fewer than half the parameters compared to ResNet. CondenseNet [57] also prunes the redundant connections between layers as seen in DenseNet. CondenseNet uses group convolutions to efficiently implement a connectivity pattern which is produced by sparsifying the network automatically during training. It uses weight pruning approach and proved to be very effective than L1 regularization throughout. This model outperformed almost all state of the art models available prior to CondenseNet and very much on par with NASNet-A on CIFAR-10 and ImageNet dataset in terms of parameter size and computation cost.

In a nutshell, the network size reduction techniques can reduce the computation overhead to a great extent, and can significantly reduce the memory footprint for the hardware. Networks such as SqueezeNet [13], MobileNets [15], and ShuffleNet [17] along with techniques like deep compression [47] provides hardware friendly optimizations in terms of resources. Comparison of the effects of pruning in terms of input size, accuracy and model size is given in the Table 2 . The effects of pruning along with quantization is shown in Table 3. AlexNet is taken as the baseline model and comparison is made by applying the pruning and quantization techniques. We can see from the tables that the model size can decreased 10-50 times with the help of pruning alone. Even though most of the above mentioned algorithms provides better optimizations in someway or another, algorithm-level optimizations do not imply the optimized hardware designs. Still works need to be done in translating these methods into hardware. Nevertheless, simplified networks helps to mitigate the hardware implementation complexity.

Table 2 Network pruning: Comparison of AlexNet model

\begin{tabular}{|l|c|c|c|c|c|}
\hline Model & $\begin{array}{c}{[13]} \\
\text { SqueezeNet }\end{array}$ & $\begin{array}{c}{[15]} \\
1.0 \text { MobileNetsV1 }\end{array}$ & $\begin{array}{c}{[15]} \\
0.5 \text { MobileNetsV1 }\end{array}$ & $\begin{array}{c}{[47] \text { Deep Compression }} \\
\text { (Pruning only) }\end{array}$ & {$[7]$ AlexNet } \\
\hline Input size & $224 \times 224$ & $224 \times 224$ & $224 \times 224$ & $224 \times 224$ & $227 \times 227$ \\
\hline Weights & $1.25 \mathrm{M}$ & $4.2 \mathrm{M}$ & $1.3 \mathrm{M}$ & NA & $61 \mathrm{M}$ \\
\hline MAC & 1700 & $569 \mathrm{M}$ & $149 \mathrm{M}$ & NA & $724 \mathrm{M}$ \\
\hline Top-1 Accuracy(\%) & 57.5 & NA & NA & NA & 57.2 \\
\hline Top-5 Accuracy(\%) & 80.3 & 70.6 & 63.7 & NA & 80.3 \\
\hline Model Size & $4.8 \mathrm{MB}$ & $16 \mathrm{MB}$ & NA & $27 \mathrm{MB}$ & $240 \mathrm{MB}$ \\
\hline
\end{tabular}

\subsection{Data-quantization and Bit-width Manipulation Techniques}

Quantization helps to reduce the number of bits required to represent the weights in a network. Bit width reduction and use of low-precision arithmetic helps to 
compress the network multi-fold and thus to reduce the memory footprint and computation requirements. From Table 3 and Table 4, we can see the advantages of quantization on different models. Networks like binarynet [61] can make use of a single bit to store the weights instead of 32-bit floating point and can drastically reduce the storage space required.

Transition from floating-point format to fixed-point format helps to reduce the area and latency. In CMSIS-NN [62], fixed-point quantization is used to optimize the neural network for resource constrained platforms like ARM Cortex-M CPUs. In this work, Fixed-point quantization uses a fixed scaling factor and is implemented as shift operations as the scaling factor used is a power of 2. A different approach of using fixed-point numbers to represent activations and floating point numbers to represent the weights in the paper [63]. The multiplication operations take a floating point number and a fixed point number as operands and mantissa multiplication is carried out by the fixed-point multiplier and exponent value is adjusted by using a shifter. Another mentionable feature in this work is the use of low precision fixed point operands for multipliers and high precision operands for accumulators, which helps to improve the accuracy. As accumulation operations are not as complex as multiplications, significant efficiency distortion won't be happening with the use of high precision accumulators. While using fixed point numbers, AlexNet and SqueezeNet requires 7 bits to achieve full accuracy while VGG-16 and GoogleNet requires 10 to 11 bits. But, the mix of floating and fixed point design which uses 3 bit mantissa and 4 bit exponent gives a normalized accuracy same as that of 7 bit and 10 bit fixed point accuracy and this involves only 3-bit multiplication. This gives a $36 \%$ reduction in memory footprint for weights and $50 \%$ reduction in power in multiplication operations.

A dynamic-precision data quantization strategy is employed by Qiu et al [64] to convert floating-point numbers to fixed point numbers with an automated quantization flow. For different layers and feature map sets, the fractional length $(f l)$ is set dynamic, and for one layer it is set static to reduce the truncation error. The analysis of this design shows that it possible to efficiently implement with shorter bit-widths using dynamic-precision quantization, which is even more efficient than static-precision quantization. The accuracy test of the models does not show any significant variation even with reduced bit-widths of 8 or 4 bits. A similar dynamic precision fixed point technique for training DNN is shown in [65, 66]. A fixed exponent won't be perfect when the range of values of activations, gradient, and parameters are different. So using different scaling factors for different variables instead of a common one can increase the efficiency. Using dynamic fixed-point numbers of bit-width 10 for propagation parameters and bit-width 12 for updated parameters shows improved error rates for MNIST and CIFAR datasets when comparing with 20 bit fixed point numbers. In Ristrett0 [66], author is more concerned about the hardware implementation strategies also. Dynamic-precision fixed point is applied for layer weights and layer outputs. For hardware implementation, the model is compressed using the Ristretto framework. This helps to compress the memory usage by 4 to 8 times than the uncompressed version. The accuracy of LeNet without compression using only 4-bit convolution and FC parameters, and 4 bit layer outputs varies only $0.4 \%$ compared to 32 -bit FP parameters, while using 2 bit convolution and FC parameters, the accuracy varies by only $1.1 \%$. 
Bit fusion [67] depicts a purely hardware-oriented optimization technique for bit-width manipulation. This work also focuses on encoding the memory-access logic efficiently by storing and retrieving the parameters with lowest possible memory footprint. The processing elements, called the bit-bricks, can reconfigure itself according to varying bit-width requirement and this architecture has its own instruction set architecture (ISA), which helps in leveraging multi-level parallelism. It can perform 1-bit, 2-bit or 8-bit operations with varying level of parallelism. Bit fusion shows much better performance and energy reduction compared to other models. A similar idea about limiting the bit-width, and utilizing on-chip and off-chip memory accesses efficiently is presented in [35]. This work proposes a technique of connecting multiple FPGAs in series using high speed serial interconnects to fit the whole network model in the on-chip memory only and proved that the speed gets doubled with serial connection. LCDet [68] follows the YOLO model for real-time object detection. LCDet replaced the last two fully connected layers in YOLO with fully-convolutional layers. Also, instead of using the floating point format, 8-bit fixed point format is used. This model achieves 4 times memory reduction and 20 times performance gain compared to the floating point model.

Binarized Neural Networks (BNN) [69], which uses binary weights and activations, is efficient in reducing the memory bandwidth without much of a reduction in accuracy. BNNs can also improve the throughput in FPGAs compared to GPUs. Yinxing Li et al [70] has shown that BNNs can actually help FPGAs to outperform GPUs despite having low operation frequency. In Binarynet [61], a method was put forward to train BNNs at run-time and computing parameters gradients at train time. While training, both the weights and the activations are set to either +1 or -1 which gives an advantage for the hardware design. Another advantage of BNNs is that the arithmetic operations can be replaced with bitwise operations and this reduces the complexity in area and time and hence increases the power efficiency. BNN implementation of a binary matrix multiplication kernel on GPU on MNIST dataset gave 7 times faster results without any loss in accuracy compared to the implementation with an un-optimized kernel. Similar works based on the approach of BNNs are proposed in Embedded Binarized Neural Networks (eBNN) [71], LightNN [72], and XNOR-Net [73]. eBNN re-orders the layer to minimize the memory used for storing intermediate results between layers in feed-forward inference. This reordering is achieved by the use of fused convolutional blocks. By this approach, the final result after pooling, batch normalization, and binary activation can be stored as a single binary digit. So, the number of floating point temporaries for storing intermediate results can be minimized and hence a $32 \mathrm{x}$ memory reduction can be achieved. eBNN gives the same accuracy as BNNs, at the same time consumes only very less memory. It takes only $3 \%$ of the overall memory to store temporaries. LightNN optimizes hardware implementation based on the BNN by replacing the multiplications by shifts or constrained shifts and add.

XNOR-Net [73] is based on BNNs and proposes two approximations to CNNsBinary weight networks and XNOR Networks. Weights of filters are binary tensors in Binary weight network, whereas in XNOR Network, both weights and inputs are binary tensors. Binary weight networks use binary weights during forward and backward propagation but uses high precision weights to update parameters. XNOR networks use binary convolution where each convolution operation consists 
of repeating shift operation and a dot product-which is an XNOR function. Binary convolution operation can perform $58 \mathrm{x}$ faster compared to conventional CNN. Comparing with state of the art models, binary convolutions achieved a memory reduction of $64 \mathrm{x}, 67 \mathrm{x}$ and $62 \mathrm{x}$ compared to double precision implementations of AlexNet, ResNet-18 and VGG-16. XNOR-Net lags behind in accuracy but can achieve very significant speedup and memory reduction. Dorefa-Net [74] concentrates on arbitrary bit-width in weights, activation and gradients similar to XNOR-Net and can be taken as a generalization of XNOR-Net. Unlike XNOR Net, the first and last layers are not quantized in Dorefa-Net. This work aims to target CPU, FPGA, ASIC and GPU implementations as bit level convolution implementation will be much efficient on hardware.

Quantized Neural Networks (QNN) [75] follows BNNs and uses binary weights and activation at run-time as well as at training while computing gradients. QNN applies binarization at the first layer as well as at the final FC layer by following quantization methods like linear quantization and logarithmic quantization, which significantly increase the power and computation efficiency. Similar to QNN, another quantization based approach, quantized-CNN (Q-CNN) [76] proposes a unified framework which applies quantization in both convolutional and fully connected layers to improve speed, minimize optimization error in each layer, as well as to compress the network. Minerva [46] uses quantization techniques to improve the power efficiency of the model targeted for resource constrained hardware platform. The evaluation results shown significant improvement on speed and compression over the competing models with slight drop in accuracy. The results on MNIST show 1.7x improvement in compression ratio compared to HashedNets [48] and Q-CNN with better accuracy than both. Similarly for ImageNet dataset this model achieves 4-6x speedup and 15-20x compression than the conventional model. Bitwidth manipulation techniques and quantization provides excellent optimization options for the hardware designer. The networks derived from binarized Neural networks actually have less memory requirements. Comparing with network pruning algorithms, the feasibility of quantization approaches on hardware are high. A comparison of accuracy after quantization for some of the models on ImageNet dataset is shown in Table 4. With Pruning and quantization, the model sizes has been decreased drastically as seen from Table 3.

Table 3 Comparison of AlexNet model after Pruning + Quantization

\begin{tabular}{|l|c|c|c|c|c|}
\hline Model & Input size & Datatype & $\begin{array}{c}\text { Top-1 } \\
\text { Accuracy(\%) }\end{array}$ & $\begin{array}{c}\text { Top-5 } \\
\text { Accuracy(\%) }\end{array}$ & Model Size \\
\hline [13] AlexNet(Baseline Caffe Model) & $227 \times 227$ & 32 -bit & 57.2 & 80.3 & $240 \mathrm{MB}$ \\
\hline [13] SqueezeNet (Deep Compressed([47])) & $224 \times 224$ & 8 -bit & 57.5 & 80.3 & $0.66 \mathrm{MB}$ \\
\hline [13] SqueezeNet (Deep Compressed([47])) & $224 \times 224$ & 6 -bit & 57.5 & 80.3 & $0.47 \mathrm{MB}$ \\
\hline $\begin{array}{l}\text { [47] Deep compression(on AlexNet) } \\
\text { (Pruning+Quantization+Huffman coding) }\end{array}$ & $224 \times 224$ & $5-8$ bit & 57.2 & 80.3 & $6.9 \mathrm{MB}$ \\
\hline [66] Ristretto (on SqueezeNet) & $224 \times 224$ & 8-bit & 57.1 & NA & $<2 \mathrm{MB}$ \\
\hline
\end{tabular}


Table 4 Accuracy comparison after quantization on ImageNet dataset

\begin{tabular}{|l|c|c|c|c|}
\hline Model & Input size & Bit-width & $\begin{array}{c}\text { Top-1 } \\
\text { Accuracy(\%) }\end{array}$ & $\begin{array}{c}\text { Top-5 } \\
\text { Accuracy(\%) }\end{array}$ \\
\hline$[13]$ SqueezeNet (Deep Compressed) & $224 \times 224$ & 6-bit & 57.5 & 80.3 \\
\hline$[13]$ SqueezeNet (Deep Compressed) & $224 \times 224$ & 8 -bit & 57.5 & 80.3 \\
\hline$[47]$ Deep Compression (GoogLeNet) & $224 \times 224$ & 5 -8bit & 57.2 & 80.3 \\
\hline$[66]$ Ristretto (SqueezeNet) & $224 \times 224$ & 8 -bit & 57.1 & NA \\
\hline$[66]$ Ristretto (GoogLeNet) & $224 \times 224$ & 8 -bit & 66.6 & NA \\
\hline$[66]$ Ristretto (CaffeNet) & $224 \times 224$ & 8 -bit & 56.0 & NA \\
\hline$[64]$ CaffeNet & $224 \times 224$ & 8 -bit & 53.2 & 76.6 \\
\hline$[64]$ CaffeNet & $224 \times 224$ & 16-bit & 53.9 & 77.1 \\
\hline$[64]$ VGG-16 & $224 \times 224$ & 8 -bit & 66.58 & 86.3 \\
\hline$[64]$ VGG-16 & $224 \times 224$ & 16-bit & 68.02 & 86.7 \\
\hline$[7]$ AlexNet & $227 \times 227$ & 32 -bit & 57.2 & 80.3 \\
\hline
\end{tabular}

3.3 Optimization Techniques to improve Accuracy, Execution Speed and Throughput

Along with network size reduction and Bit-width manipulation techniques, it is worth considering techniques to effectively improve accuracy and speed of the neural networks. These techniques not only improves the efficiency of the network, but also helps in a way to reduce the network size. Some of the most employed methods are discussed here.

As the neural networks go wider and deeper, the accuracy no longer becomes an issue, but the main problem will be the performance at inference as the time consumed to make the predictions will be more as well as the memory footprint requirements. Knowledge Distillation [77] provides a solution to this issue and improves the performance of deep learning models on mobile devices. The main idea behind this is to extract features by training a large complex network which can produce better predictions and then by using this model we can train a small network to produce comparable predictions or in other words transferring knowledge from a complex network to a small network. The cumbersome model can be called a Teacher Network and small model can be called a student Network. The student network is trained to mimic the output of the Teacher network rather than training with the raw data. The experiments with MNIST dataset and speech recognition experiments shows satisfying results as the distilled model produces almost the same results as the ensemble model and better results than the baseline model. FitNets [78] uses Knowledge Distillation to improve the performance of DNN implementations. The student network of FitNets is called FitNet and learning of intermediate representation is performed using the Hints from teacher. The results on benchmark datasets like CIFAR, MNIST and SVHN shows improved performance compared to the teacher model in terms of accuracy and number of parameters and nearly provide state of the art performance. Lu [79] proposes a method to apply Knowledge distillation to Highway networks [80] to optimize the model to achieve a small memory footprint. Apprentice [81] combines low-precision arithmetic and knowledge distillation to improve the performance of CNNs, which proved to be efficient considering that it can achieve state-of-the-art accuracy using ternary and 4-bit precision on ResNet architecture. 
A well-known architecture for semantic pixel-wise labelling or semantic segmentation for object detection is SegNet [82]. SegNet uses an encoder-decoder architecture where thirteen VGG-16 convolution layers are used as the encoder and Up-samplers to each convolution layer is used as the decoder. The encoder uses subsampling along with max-pooling to improve the classification accuracy but this reduce the input feature size. To increase the feature map size, up-sampler is used as the decoder and it stores only max-pooling indices for decoding purpose which saves memory space with slight loss in precision. SegNet was able to reduce the number of parameters significantly from 134M (VGG) to $14.7 \mathrm{M}$ by avoiding the FC layer as the decoder.

Table 5 Performance comparison of some of the most efficient networks on ImageNet Dataset

\begin{tabular}{|c|c|c|c|c|c|}
\hline Model & Image Size & Parameters & MAC & $\begin{array}{c}\text { Top-1 } \\
\text { Accuracy }(\%)\end{array}$ & $\begin{array}{c}\text { Top-5 } \\
\text { Accurcay }(\%)\end{array}$ \\
\hline [9] Inception V1 & $224 \times 224$ & $6.6 \mathrm{M}$ & $1448 \mathrm{M}$ & 69.8 & 89.9 \\
\hline [26] Inception V2 & $224 \times 224$ & $11.2 \mathrm{M}$ & $1940 \mathrm{M}$ & 74.8 & 92.2 \\
\hline $\begin{array}{ll} & \text { Inception V3 }\end{array}$ & $299 \times 299$ & $23.8 \mathrm{M}$ & $5720 \mathrm{M}$ & 78.8 & 94.4 \\
\hline [54] Inception-ResNet-V2 & $299 \times 299$ & $55.8 \mathrm{M}$ & $13200 \mathrm{M}$ & 80.1 & 95.1 \\
\hline [83] SENet & $320 \times 320$ & $145.8 \mathrm{M}$ & $42300 \mathrm{M}$ & 82.7 & 96.2 \\
\hline [27] Xception & $224 \times 224$ & $22.8 \mathrm{M}$ & $5700 \mathrm{M}$ & 79.2 & 94.5 \\
\hline [84] ResNeXt-101 & $320 \times 320$ & $83.6 \mathrm{M}$ & $31500 \mathrm{M}$ & 80.9 & 95.6 \\
\hline 21] NASNet-A $(\mathrm{N}=5, \mathrm{~F}=1538)$ & $299 \times 299$ & $10.9 \mathrm{M}$ & $2350 \mathrm{M}$ & 78.6 & 94.2 \\
\hline [15] 1.0 MobileNet-224 & $224 \times 224$ & $4.2 \mathrm{M}$ & $569 \mathrm{M}$ & 70.6 & 89.5 \\
\hline 17] ShuffleNet 2x & $224 \times 224$ & $5 \mathrm{M}$ & $524 \mathrm{M}$ & 70.9 & 89.8 \\
\hline [19] MobileNetV2 & $224 \times 224$ & $3.4 \mathrm{M}$ & $300 \mathrm{M}$ & 72.0 & NA \\
\hline 21] NASNet-A $(\mathrm{N}=4, \mathrm{~F}=44)$ & $224 \times 224$ & $5.3 \mathrm{M}$ & $564 \mathrm{M}$ & 74.0 & 91.6 \\
\hline 24 AmoebaNet-A(N=4, F=50) & $224 \times 224$ & $5.1 \mathrm{M}$ & $555 \mathrm{M}$ & 74.5 & 92.0 \\
\hline 24 AmoebaNet- $\mathrm{C}(\mathrm{N}=4, \mathrm{~F}=50)$ & $224 \times 224$ & $6.4 \mathrm{M}$ & $570 \mathrm{M}$ & 75.7 & 92.4 \\
\hline$[22]$ PNASNet- $5(\mathrm{~N}=3, \mathrm{~F}=54)$ & $224 \times 224$ & $5.1 \mathrm{M}$ & $588 \mathrm{M}$ & 74.2 & 91.9 \\
\hline [21] NASNet-A $(\mathrm{N}=6, \mathrm{~F}=168)$ & $331 \times 331$ & $88.9 \mathrm{M}$ & $23800 \mathrm{M}$ & 82.7 & 96.2 \\
\hline [24] AmoebaNet-A(N=6, $\mathrm{F}=228)$ & $331 \times 331$ & $155.3 \mathrm{M}$ & $41100 \mathrm{M}$ & 83.1 & 96.3 \\
\hline \begin{tabular}{l|l}
{$[22]$} & PNASNet-5 $(\mathrm{N}=4, \mathrm{~F}=216)$
\end{tabular} & $331 \times 331$ & $86.1 \mathrm{M}$ & $25000 \mathrm{M}$ & 82.9 & 96.2 \\
\hline [57] CondenseNet $(\mathrm{G}=\mathrm{C}=4)$ & $224 \times 224$ & $2.9 \mathrm{M}$ & $274 \mathrm{M}$ & 71.0 & 90.0 \\
\hline [57] CondenseNet $(\mathrm{G}=\mathrm{C}=8)$ & $224 \times 224$ & $4.8 \mathrm{M}$ & $529 \mathrm{M}$ & 73.8 & 91.7 \\
\hline [25] DARTS & $224 \times 224$ & $4.9 \mathrm{M}$ & $595 \mathrm{M}$ & 73.1 & 91.0 \\
\hline [23] MnasNet-92 & $224 \times 224$ & $4.4 \mathrm{M}$ & $388 \mathrm{M}$ & 74.79 & 92.05 \\
\hline [23] MnasNet-65 & $224 \times 224$ & $3.6 \mathrm{M}$ & $270 \mathrm{M}$ & 73.02 & 91.14 \\
\hline [23] MnasNet-92+SE & $224 \times 224$ & $5.1 \mathrm{M}$ & $391 \mathrm{M}$ & 76.13 & 92.85 \\
\hline [23] MnasNet-65+SE & $224 \times 224$ & $4.1 \mathrm{M}$ & $272 \mathrm{M}$ & 74.62 & 91.63 \\
\hline
\end{tabular}
MAC-Multiply-accumulate

Most of the techniques to improve the performance adversely affect the hardware implementation. A few of techniques like knowledge distillation and semantic segmentation might help to reduce the complexity while keeping the performance. However, translating such techniques on to hardware is still a hard task to perform and efficient hardware frameworks for such tasks is a real necessity. A comparison of some of these optimized models with the state-of-the-art models in terms of parameter size, MAC operations, and accuracy is shown in Table 5. This would a fair idea about the complexity of the networks while considering for inference on hardware. 
3.4 Automated or Neural architecture search based Optimization Techniques

Manually creating efficient models is a very complex task and hence there is a lot of focus on creating an automated approach of neural network design. Such an unsupervised approach gave rise to some of the most efficient architectures till date such as NASNet [21], PNASNet [22], AmoebaNet [24], DARTs [25] and MNASNet [23]. Neural architecture search (NAS) framework based architecture NASNet is the first automated model proposed by Google. The key feature of this work is NASNet search space which helps to generate an architecture for a large dataset by using a small dataset using reinforcement learning search method as in NAS framework. To create a scalable architecture for any image size, two types of convolutional cells are used. One is the Normal cell-which returns the same size feature map and other is the reduction cell which returns a feature map having width and height reduced by 2 . These cells are stacked in feed forward nature to a desired number to suit the size of the dataset to be trained to create the CNN architecture. In this work, the model is trained with CIFAR-10 dataset and then it is scaled for ImageNet dataset. Comparison of performance on ImageNet dataset compared to models like Inception-V2, Inception-V3 [26], Inception-ResNet-V2 [54], Xception [27], ResNeXt [84] and SENet [83] shows improved top-1 and top-5 accuracies with lower parameter and computation cost. Also, NASNet tops in accuracy in comparison with models which uses less than 600M MAC operations like MobileNet [15] and ShuffleNet [17]. A detailed comparison of these models is given in Table 5 in terms of accuracy and parameter size.

PNASNet [22] is a modified and improved version of NASNet. The basic structure is based on NASNet, but have reduced the number of available functions and combinational operators and hence was able to reduce the training time 5 times with the same accuracy. Unlike NASNet, PNASNet uses a progressive Neural architecture Search in which the reinforcement learning is replaced by a sequential model-based optimization (SMBO) strategy. The evaluation on CIFAR-10 dataset shows 21 times less computation compared to NASNet. PNASNet shows slightly better performance than NASNet on ImageNet dataset under mobile setting, while we can say that both networks are at par on accuracy for large models.

Reinforcement learning and evolutionary algorithms for search space, a different approach compared to NASnet and AmoebaNet is used in DARTs [25]. It is based on the continuous relaxation of the architecture representation which allows efficient search of the architecture using gradient descent. This method reduces the training time by a huge margin. This algorithm is applicable to both convolutional and recurrent neural networks. In comparison, Darts is slightly below NASNet-A in accuracy on CIFAR-10 dataset but the search cost is $450 \mathrm{x}$ less than NASNet.

One disadvantage with models based on NAS is the large search cost. The main reason behind this is that while training the child architecture generated by the search space, only accuracy is measured and the weights are not reused. ENAS [85] subsides the problem of large search cost by sharing weights in child models. In ENAS, NAS's search space is represented by a single directed acyclic graph (DAG) and all the child models can be modeled as sub-graphs of this DAG. In DAG, the nodes represent local computations and edges represents the flow of information. The nodes have its own parameters and used only when a computation is activated 


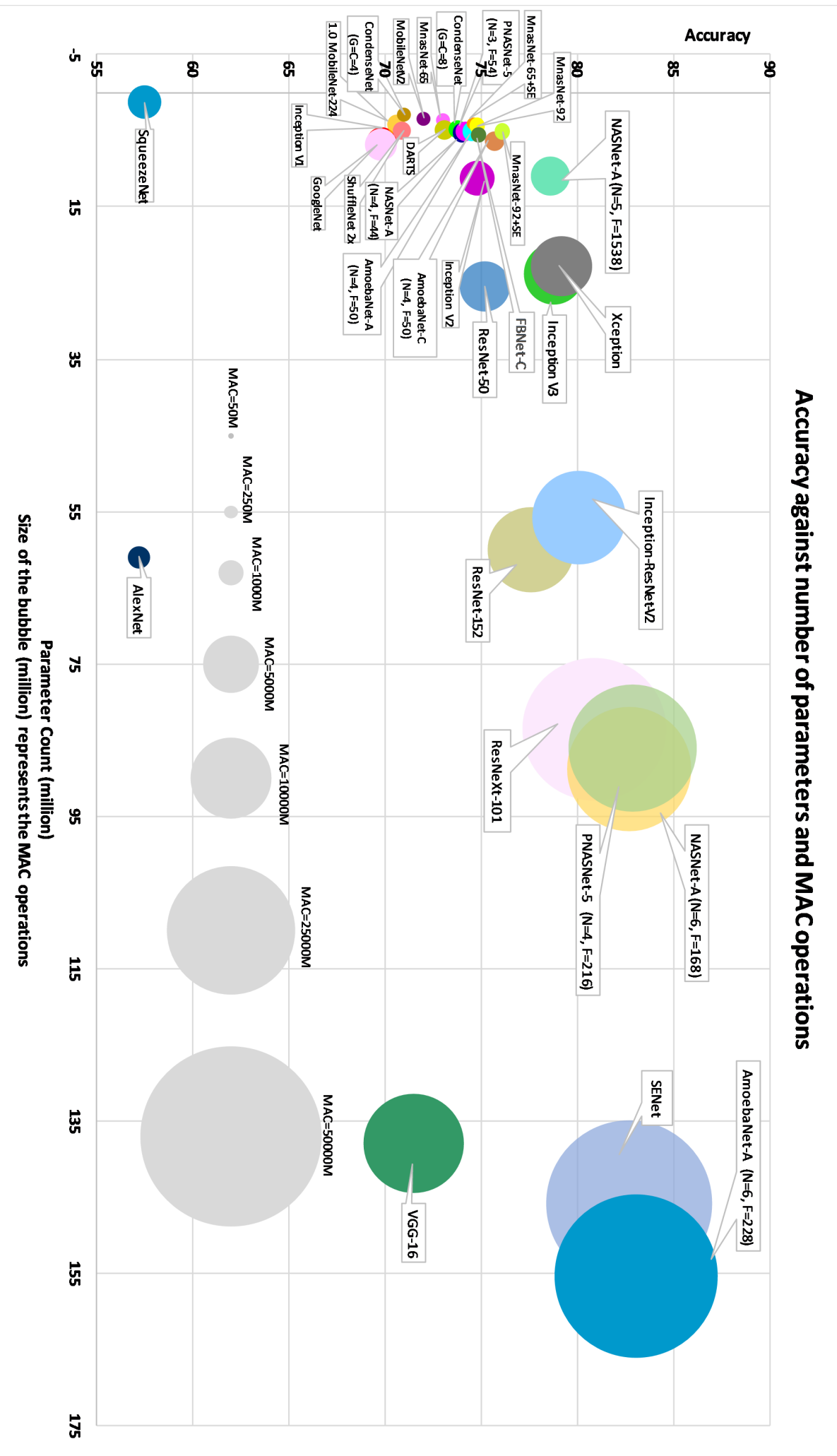

Fig. 2 Accuracy against number of parameters and MAC operations 
and these parameters can be shared among all child models. This considerably reduces the search time. Compared to NASnet, ENAS is almost 4000x faster in search cost with almost comparable accuracy. Hardware aware architectures such as MnasNet and AmoebaNet are discussed in detail in section 5.

A more detailed comparison of these models with other state of the art models is provided in Table 5. In Table 5, all the models which use ImageNet dataset for evaluation are included. Figure 2 shows a comparison of accuracy against parameter count and MAC operations of various models.

\section{Hardware-Specific Optimization Techniques on Various Platforms}

Hardware architecture optimization is essential for real-time inference acceleration of deep learning models because of its strict latency requirements [86]. Algorithmic optimizations help to reduce the complexity of the network, whereas hardware optimization techniques help to provide similar performance of an implementation in a constrained device. Most of the complex algorithmic optimizations are not feasible to translate directly on to FPGA. Hence, finding practical approaches to transfer the algorithms on hardware is of great importance. Though this study targets FPGAs mainly, there has been significant work by the systems communities to address the challenges of compiling CNNs to resource constrained platforms other than FPGAs. This not only include the hardware implementations but also the libraries and frameworks which helps to make the life easier for the hardware designer. These works are equally important in inference on FPGAs. Optimization techniques for FPGAs and other hardware platforms are described in the following sections. Section 4.1 focuses on some of the works CPU, GPU, ASIC, and Embedded Processor based systems and section 4.2 focuses mainly on FPGA based systems.

\subsection{Optimization techniques on CPU, GPU, ASIC, and Embedded Processor} based systems

CPU alone systems are not really suitable for training and inference of deep neural networks because of their limited ability to handle the computational complexity and resource requirements [30,62]. There are a few works available which shows how to effectively handle the requirements of deep neural networks on CPUs and these techniques are very much suitable for FPGAs also.

\subsubsection{Architectural optimizations}

The architectural optimizations targeting general putpose processors is more generic and could be easily adapted to FPGAs. Some of the works targeting generic hardware optimizations are discussed in this section. A well optimized hardware accelerator design aimed for better optimizations based on memory, performance and energy on an ASIC platform is DianNao [87]. Naive form of hardware implementation is not scalable and is suitable only for small neural networks. In DianNao, the accelerator consists of a control logic (CP), computational block called Neural Functional Unit (NFU) to which an input buffer for input neurons 
(NBin), an output buffer for output neurons (NBout), and a third buffer for synaptic weights (SB) are connected. A staggered pipeline is used in NFU and instead of using 32-bit floating point operators, the arithmetic operations use 16-bit fixed point operators. The control logic uses control instructions to explore different layer implementations like partitioning and scheduling. The accelerator can provide a throughput of $452 \mathrm{GOP} / \mathrm{s}$ within a small footprint of $3.02 \mathrm{~mm} 2$ and $458 \mathrm{~mW}$. Compared to a SIMD core, the proposed accelerator is on an average $117.87 \mathrm{x}$ faster. One drawback of this accelerator is the high energy consumption required for memory access which makes this accelerator design fall behind some of the competing models even though it gives a better energy ratio compared to SIMD. DaDianNao [88] is introduced to eliminate the drawbacks in memory bandwidth requirements of DianNao [87]. DaDianNao has a fully distributed architecture in which the synapses are stored close to the neurons which uses them. This minimizes data movement and hence saves energy and time. Each node footprint is massively biased towards storage rather than computations. The general architecture of DaDianNao has a set of identical nodes arranged in a mesh topology. Each node contains a neural computational unit-NFU and storage. The NFU is basically as in DianNao model, but much more complex and can be reconfigured for each layer.

A highly automated co-design approach to optimize hardware accelerators which aims optimization not only on the hardware aspect, but also across the algorithm, architecture, and circuit levels is described in Minerva [46]. Minerva's automated design flow consists of five stages. The first two stages build a fine baseline micro-architecture of the design by exploring the DNN training and micro architectural design spaces of accelerator. These two stages are then followed by 3 cross-layer optimization stages. The third stage uses data type quantization to independently modify the precision and range in each layer. The selective operation pruning stage identifies the insignificant neurons whose values are close to zero and eliminate them. The SRAM fault mitigation stage proposes new fault mitigation techniques by identifying the SRAM read faults and then rounding the faulty weights to zero. These optimization stages can reduce the power consumption by $8 x$. Even though the model shows mixed performance improvements with competing models, the reduction in power consumption with comparable error rate is the main advantage of this design.

An accelerator architecture Eyeriss [89] was proposed to deliver state of the art performance with minimum energy consumption. The key feature of this architecture is an efficient CNN data flow called row stationary (RS) which exploits the data reuse and minimizes the data movement and it can adapt to different CNN shapes. The architecture exploits the sparsity of the network and executes data operations only on non-zero values and compresses the data to reduce data movement. An analysis framework is also modeled to improve the energy analysis efficiency. The ASIC implementation details given in [89] shows a maximum throughput of 16.8-42 GOPs with a frame rate of 34.7 FPS for convolutional layers on AlexNet model using 16-bit fixed word length. Eyeriss-V2 [90] is introduced with a new data flow Row Stationary Plus ( $\mathrm{RS}+$ ) along with a low cost scalable NoC design called hierarchical mesh, and a performance analysis framework called Eyexam. This model claims to support on-chip data delivery for high data reuse and high bandwidth applications. Compared to Eyeriss V1, Eyeriss V2 achieves an average speed up of 2082.6x, 757x and 1083.2 for AlexNet, GoogLeNet and 
mobilenet respectively while evaluating for 16383 PEs with a maximum obtained speedup of 4096x for all these networks.

A design for implementing such networks in CPU for $\mathrm{x} 86$ processors is presented in [30]. Both fixed point and floating point implementation techniques are employed in this work. Proper optimized data layout for SIMD, and use of Streaming SIMD Extensions 2 (SSE) instruction for floating point SIMD are taken in to consideration and optimized to improve the performance. For fixed point implementation, linear quantization is performed along with the use of SSE3 and SSE4 instructions. The system shows 10x improvement against the baseline floating point implementation and $4 \mathrm{x}$ improvement against the best floating point system. Performance optimization methods for Neural Networks on resource-constrained microcontroller platforms is explained in CMSIS-NN [62] using ARM Cortex-M CPU as the target platform. It also uses fixed point quantization as explained in section 3.2 along with efficient software kernels for data transformation and for each neural network layers.

\subsubsection{Translation of Algorithmic optimizations}

Mapping of algorithmic optimizations onto hardware is of utmost important and such techniques must be studied thoroughly. Translation of such algorithmic optimizations on generic hardware is discussed in this section.

Even though CPUs are not a good choice as standalone processing units in High performance computing systems, heterogeneous computing systems with the association of GPUs can offer high performance by leveraging parallelism and high memory bandwidth. A few of such implementation techniques on GPU based systems can be adapted to FPGAs as well because of its parallelism exploting strategies and energy efficiency. The work in [91] proposes a convolution method targeting GPUs and this work uses Winograd algorithm for performing convolution. The main features of [91] is ZeroSkip hardware mechanism which is used to avoid the redundant zero weights, and AddOpt mechanism-which is used to eliminate the limitation of Winograd algorithm by data reuse. The experimental results show that the combination of ZeroSkip and AddOpt gives the best execution time compared to the basic version. Also this combination gives an energy reduction of $50.2 \%$.

An ARM processor based design in [34] put forward an argument that instead of optimizing the hardware alone, the scope of optimization must be the entire SoC, not just the accelerator. This paper shed light to mainly 3 areas of SoC optimization namely Accelerator Interfacing, Software Abstractions and System optimizations. While interfacing an accelerator, the cache/memory interface between the accelerator and the SoC is critical. To achieve this, the authors suggests to leverage the L3 cache in the CPU cluster as a bandwidth filter rather than directly interfacing the NNs with the DRAM. Also, ARM provide optimized implementations for DNN kernels in the form of ARM Compute Library. The whole system optimization is also important which can exploit new opportunities for optimization which won't be visible when we consider only a part of the solution.

EIE (energy efficient inference engine) [92] is An accelerator on ASIC for sparse vector matrix multiplication for performing inference on the deep compressed network model described in [47]. EIE is a well optimized model which uses Deep 
Compression technique [47] and weight sharing for hardware implementation. EIE enables large compressed networks to fit into SRAM than DRAM, which results in 120x energy reduction compared to DRAM. Also, Exploiting sparsity of activation and avoiding zero activations gives approximately $65 \%$ energy reduction. On the hardware, distributed storage and distributed computation parallelize the sparse layer across multiple Processing Elements (PEs) and this results in better scalability and load balance. In almost every details like load efficiency, scalability and execution time of layers, EIE outperforms other implementations by a huge margin. Comparisons of these models with other existing works are shown in Table 6 .

DeepRebirth [93] is an acceleration strategy for neural networks targeting mobile platforms like Samsung Galaxy S5, Moto E, Samsung Galaxy S6 (all these use ARM CPU architecture), Macbook Pro (uses Intel x86 architecture) and Titan $\mathrm{X}$ GPU. The key feature in this work is the merging of non-tensor layers with its neighbouring layers without affecting the functionality by re-training the newly generated parameters. Two methods namely streamline slimming and branch slimming are used to achieve this. This model can be applied to any network to compress that network. The slimmed deepRebirth version of GoogleNet with tucker decomposition outperforms other versions of GoogleNet and SqueezeNet in mobile platforms, whereas SqueezeNet shows better performance in GPU.

Implementaions on processor based systems concentrates more on area and energy optimizations compared to FPGA based designs. Implementation techniques such as memory sharing and data resuse, fixed-point implementations [30,62], Optimization as a whole [34], partitioning and scheduling $[87,89]$, and reducing sparsity $[89,92,94]$ can be adopted efficiently to FPGA-based platforms. A record of some of the implementations on different platforms is given in Table 6. A fair comparison of these models is not possible as different models use different networks and datasets for evaluation.

Table 6 Implementation details and comparison of CPU \& ASIC implementations

\begin{tabular}{|l|c|c|c|c|c|c|c|c|c|}
\hline Model & Platform & $\begin{array}{c}\text { Frequency } \\
(\mathrm{MHz})\end{array}$ & $\begin{array}{c}\text { Maximum } \\
\text { Model size } \\
\text { (parameters) }\end{array}$ & Precision & $\begin{array}{c}\text { Area } \\
(\mathrm{mm} 2)\end{array}$ & $\begin{array}{c}\text { Power } \\
\text { (W) }\end{array}$ & $\begin{array}{c}\text { Throughput } \\
\text { (Images/sec) })\end{array}$ & $\begin{array}{c}\text { Area } \\
\text { Efficiency } \\
\text { (Images/ } \\
\text { s/mm2) }\end{array}$ & $\begin{array}{c}\text { Energy } \\
\text { Efficiency } \\
\text { (Images/J) }\end{array}$ \\
\hline$[92]$ Core-i7 5930K & CPU & 3500 & $<16 \mathrm{G}$ & 32 -bit float & 356 & 73 & 162 & 0.46 & 2.22 \\
\hline$[92]$ GeForce Titan X & GPU & 1075 & $<3 \mathrm{G}$ & 32 -bit float & 601 & 159 & 4115 & 6.85 & 25.9 \\
\hline$[92]$ Tegra K1 & mGPU & 852 & $<500 \mathrm{M}$ & 32 -bit float & NA & 5.1 & 173 & NA & 33.9 \\
\hline$[64]$ & FPGA & 150 & $<500 \mathrm{M}$ & 16 -bit fixed & NA & 9.63 & 33 & NA & 3.43 \\
\hline$[8]$ & ASIC & 606 & $18 \mathrm{M}$ & 16 -bit fixed & 67.7 & 15.97 & 147938 & 2185 & 9263 \\
\hline$[92]$ & ASIC & 800 & $84 \mathrm{M}$ & 4-bit fixed & 40.8 & 0.59 & 81967 & 2009 & 138927 \\
\hline$[92]$ & ASIC & 1200 & $336 \mathrm{M}$ & 4 -bit fixed & 63.8 & 2.36 & 426230 & 6681 & 180606 \\
\hline$[94]$ (VGG) & ASIC & 500 & NA & $16-32$ bit fixed & 6.3 & 0.257 & 13.71 & 2.17 & 53.34 \\
\hline$[90]$ (Alexnet) & ASIC & $100-250$ & NA & 16 -bit fixed & 12.25 & 0.278 & 34.7 & 2.83 & 124.82 \\
\hline NA-Not Available
\end{tabular}

\subsection{Optimization techniques on FPGA-based Systems}

Despite the fact that the association of GPUs with CPUs can leverage high floating point performance, most GPUs are power hungry and hence are less favorable for embedded applications. High parallelism and flexibility along with better power efficiency give FPGAs an edge over GPUs. Optimization techniques employed on FPGAs for implementing CNNs are discussed in this section. 


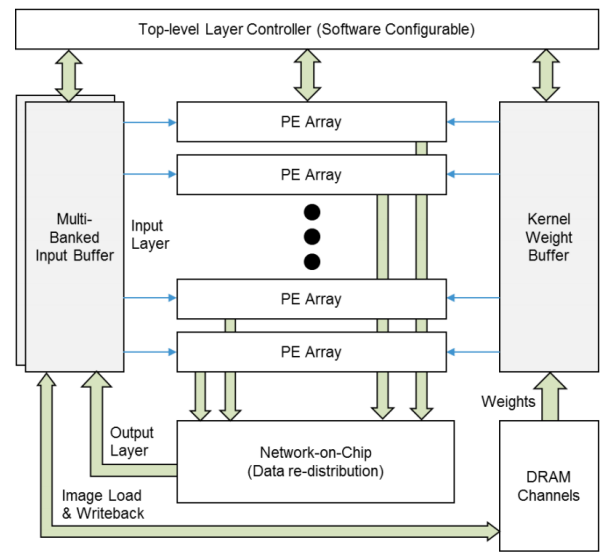

Fig. 3 Hardware architecture based on Catapult FPGA [95]

\subsubsection{Architectural optimizations}

The hardware architectures of the FPGA acclerators follow the same strtucture where the difference is being the processing engines, communication, and memory sharing options. The architectural optimizations of some the recent works are discussed in this section.

The Catapult project $[1,95]$, which is a reconfigurable fabric logic designed for datacenters and was evaluated for Bing search engine, has successfully shown the ability of FPGAs to accelerate neural networks in datacenters. Following the Catapult design, Microsoft research team implemented a high throughput CNN accelerator on StratixV D5 FPGA consisiting of multi-banked input buffers, kernel weight buffers and on-chip data re-distribution network which can minimize the off-chip memory access. This model shows $2.9 x$ throughput over [29] and $1.5 x$ and $3.35 \mathrm{x}$ power efficiency over caffe + cuDNN implementation on Tesla K40 and Tesla K20 GPUs respectively over ImageNet $1 \mathrm{k}$ dataset. The hardware architecture of the design is shown in figure 3 .

As one of the main challenges in FPGA based design is the memory bottleneck, [96] gives a fair idea about memory-centric approach of FPGA implementation of CNN using HLS. Since most of the workload is in the feature extraction layers, the algorithm merges the convolution and subsample layers to reduce the computation workload. Flexible reuse buffers implemented with FPGA block RAMS are used for the MAC PEs to effectively utilize the data reuse for predictable data access pattern. The microblaze processor is used to control the operations. Optimized scheduling and pipelined Tile processing is used in the design flow to increase the efficiency. By using such a design flow, it shows that the FPGA resources can be reduced up to $13 \mathrm{x}$ compared to other accelerators with standard scratchpad memories.

ConvAU [32] is an FPGA based CNN accelerator architecture which uses a systolic array which is a homogeneous grid of processing elements (PEs). PEs are multiply and accumulate (MAC) computation units, with each element connected only to its neighboring PEs. PEs take inputs from the neighbouring PEs and the result is stored in the local memory. The data is fed to PEs using a 256x256 

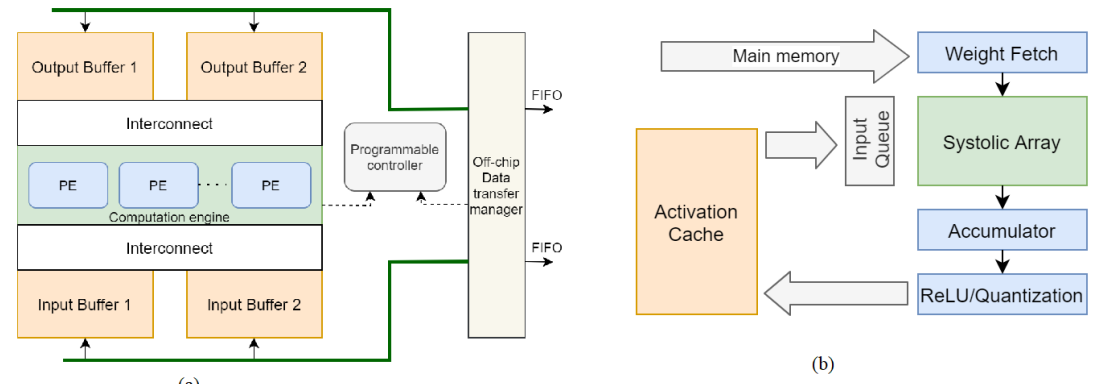

(b)

Fig. 4 Hardware architectures based on: (a) Roofline model [29] (b) systolic array model [32]

8-bit SRAM. A uniform quantization with static representations is used for matrix multiplications with 8 bit multipliers and 32-bit accumulators. The activation function used is ReLU. Pipelining methods are employed for weight loading, matrix multiplication and ReLU activation. Even though ConvAU can provide better results than Tensor Processing Unit (TPU) [97] for the ImageNet dataset, the comparison given in the paper is not a fair one because ConvAU uses SqueezeNet which has a model size of only $4.8 \mathrm{MB}$ on on-chip memory whereas TPU has an on-chip memory size of 28MB. Another FPGA-based systolic array architecture for CNN computation acceleration by making use of stochastic rounding method is proposed in [31].

An analytical design scheme using the roofline model is discussed in [29]. The design aims for the best performance with lowest FPGA resource requirement by quantitatively analyzing the computing throughput and required memory bandwidth of the model with the help of various optimization techniques for computation optimization like loop unrolling, loop pipelining and Tile size selection. To optimize the memory access, techniques like local memory protection and loop transformation for memory reuse are used. The implementation results shows reduced wastage of FPGA resources.

A general hardware architecture employing roofline model [29], and systollic arrays [32] is shown in figure 4 (a) and (b) respectively.

The work done in [98] introduces an approach to accelerator design by dividing FPGA resources to multiple processors. Such a design can work on multiple images in parallel and can reduce the computation time while using the same resources. The authors also propose an optimization algorithm which can partition the FPGA resources into multiple convolutional layer processors (CLP) given the resource budget. CLP modeling is performed based on cost, performance and memory usage and then optimized for computation and memory. The evaluation results on Virtex-7 FPGAs for AlexNet, VGGNet-E, SqueezeNet and GoogLeNet clearly shows that the percentage utilization of resources is in the high nineties for multi-CLPs for both fixed and floating point, whereas for single-CLP it can go as low as $23.7 \%$ for fixed point and $65.4 \%$ for floating point. Also Multi-chip design shows high throughput even though it requires more BRAM usage. The basic S-CLP design model used for comparison is adapted from [29]. Bit Fusion [67] also targets FPGAs for CNN implementation which makes use of bit-width manipulation techniques and it is discussed in section 3.2. 


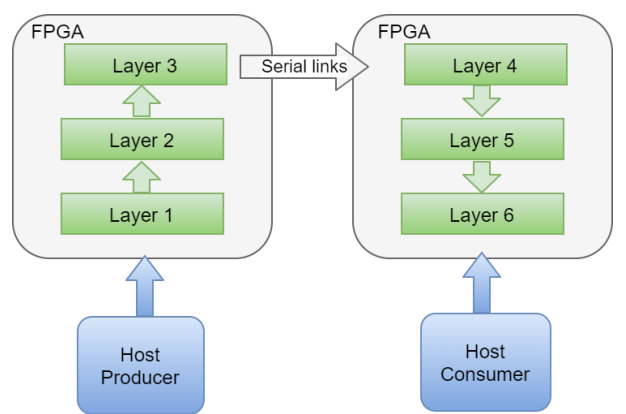

Fig. 5 Multi-FPGA architecture [35]

\subsubsection{Translation of Algorithmic optimizations}

The efficient mapping of algorithmic optimizations to FPGA is discussed in this section.

An FPGA accelerator using a systematic design space methodology is presented in [36]. The design space exploration model does a performance modeling after formulating the optimization problem followed by resource utilization modeling and optimization of the OpenCL framework modules. The operations use 8-16-bit fixed point precision and the fixed point implementation on OpenCL compared to full precision Caffe model showed a $2 \%$ decrease in accuracy. However,the FPGA implementation shows a maximum of $9.5 \mathrm{x}$ and $5.5 \mathrm{x}$ speedup for AlexNet and VGG respectively compared to Caffe CPU implementation. A CNN accelerator design using OpenCL on Altera FPGAs is described in [35]. It integrates Caffe framework to provide a high-level network description. While implementing $\mathrm{CNN}$, the inner product layers consume less computational time than the convolutional layers and hence need less parallelization and can be moved to host CPU. This helps in balancing the layers in such a way that all the layers can be computed in the same amount of time. The multiplications are done in FPGA logic and hence to increase the performance, the number of multipliers is increased by reducing the bit width of the multiplier. Since all computations in convolution layers can be computed in parallel, this increases the external memory bandwidth required for loading layers. To reduce memory bandwidth, all static parameters can be stored in the on-chip memory and if on-chip memory is not sufficient for a single FPGA, multiple FPGAs can be connected together using high-speed serial interconnects and this will also speed up the whole model. The architecture using multiple FPGAs is shown in figure 5 .

The effectiveness of employing Winograd algorithm is proved in [99] and [100]. An OpenCL architecture named as Deep Learning Accelerator (DLA) to accelerate CNN on Arria 10 FPGAs is proposed in [99]. Winograd transform is employed for multiply-accumulate operations in this work. Winograd's minimal filter algorithms can be adapted when the convolution stride is 1 and this can minimize the complexity of the arithmetic operations. Networks like GoogleNet, ResNet and other deeper derivations of these networks uses small filter and hence Winograd's algorithm is very much favorable for such networks. The processing elements use stream buffers to store the intermediate features on the on-chip memory 
and this helps to minimize the bandwidth requirements of convolution and fully connected layers in a much better way than the batching methods. Half precision floating point format is used to reduce the resource requirement. For AlexNet implementation, in comparison with prior works on GPU and FPGA, this model provides 1382 GFLOPs which is $8.4 \mathrm{x}$ and 19x speedups in terms of GFLOPs against KU060 GPU and Stratix V FPGA respectively. Also, this work can provide comparable performance against GPUs like TitanX and M4 in terms of normalized power consumption (img/s/W). A better approach using Winograd transform using 16-bit fixed point format and line buffers for data reuse is proposed in [100]. This work shows 10x improvement in throughput compared to other works on FPGA and 3x better performance compared to GPU.

Work on improving the performance of CNNs on FPGAs by optimizing the memory bottleneck is discussed in [101]. This work is based on the assumptions that the applications of interest are bandwidth bounded, not latency, and the speed of the network is dependent on the slowest path in the memory hierarchy. Hence it will be a better approach to coalesce the external memory access to achieve maximum streaming bandwidth. The design is based on a proposed performance balance model. Based on the model analysis performed on the basis of parameters like machine balance $B m$, which is the balance between memory bandwidth, computational performance and code balance $B c$ which is the memory bandwidth requirement of the OpenCL kernel, the kernel design is made. The design methodology proposes a two-dimensional multi-cast interconnection between the PEs and local memory which helps to reuse the data from on-chip BRAM by multiple PEs.

A design flow for embedded FPGA platforms for object detection is introduced in [102]. This design is based on an optimization flow having network architectures and resource constraints as inputs and then look for generating the best performance model by tuning the available hardware parameters. The hardware architecture of the model consists of two kernels namely conv kernel and FC kernel. These kernels consists of input and output buffers which helps in data reuse and computation kernel which performs the convolution and matrix multiplication operations. The conv kernel runs parallel convolution computation kernels. Given the CNN parameters, the design model of the architecture can predict the resource usage and this will help to formulate insights into the optimization of the model using these parameters. The implementation on FPGA for YOLO and Fast RCNN gives an overall execution time of $0.744 \mathrm{~s}$ and $0.875 \mathrm{~s}$ respectively which are negligible compared to the best software performance on ARM architecture which is 36.92s. The GPU implementation is faster but the power efficiency in FPGA is 26.5x better than in GPU. Sateesan et al. [103] presents a similar design technique targeting resource constrained devices exploiting the pipelining and parallelism. This work also employs optimized kernels to reduce the resource utilization, and to improve the performance.

The work in [104] exploits the inefficiencies in conventional multipliers and proposes Pragmatic (PRA) architecture to improve the speed and energy efficiency of CNN convolutional layer computations. PRA calculates only non-zero terms in multiplication unlike the conventional bit-parallel multipliers and also uses reduced precision for operators. This model achieves $2.6 \mathrm{x}$ performance and $28 \%$ energy efficiency compared to DaDiaNao [88] even with an 8-bit quantized representation. Sparse CNN (SCNN) [105] is similar in a way to PRA [104], exploits the zero-valued 
weights and activations as a result of pruning and ReLU operations. SCNN make use of a novel dataflow which eliminate the unnecessary data transfers and reduce storage requirements by exploiting the sparsity.

A sparse matrix compression algorithm to exploit the sparsity of the neuron activation in CNN and thus to reduce the memory footprint for FPGA accelerator implementation is proposed in NullHop [94]. The NullHop accelerator architecture has a Compute Core Module (CCM) which accepts inputs from the on-chip SRAM. The input feature maps for each layer and kernels are stored in off-chip DRAM. Output feature maps of each layer is offloaded to DRAM in a compressed format. The input feature maps for CCM are transferred from DRAM to SRAM. The Input data processor (IDP) of the NullHop architecture performs the operation of storing feature maps for CCM and decoding the compressed feature maps. The CCM is followed by ReLU and pooling units. The implementation is done on Xilinx Zynq $7100 \mathrm{SoC}$ and tested for real-time applications by interfacing to a neuromorphic event camera.

Table 7 Comparison of CNN implementations on FPGA

\begin{tabular}{|l|l|l|c|c|c|c|c|}
\hline Model & FPGA Platform & Frequency & Precision & $\begin{array}{c}\text { Performance } \\
\text { (GOPs/s) }\end{array}$ & $\begin{array}{c}\text { Performance } \\
\text { Density } \\
\text { (GOPs/DSPs/ } \\
\text { cycle) }\end{array}$ & $\begin{array}{c}\text { CNN size } \\
\text { (GOPs) }\end{array}$ & $\begin{array}{c}\text { Power } \\
\text { Efficiency } \\
\text { (GOPs/s/W) }\end{array}$ \\
\hline$[96]$ & Virtex6 VLX240T & $150 \mathrm{MHz}$ & Fixed point & 17 & 0.15 & 5.48 & $\mathrm{NA}$ \\
\hline$[29]$ & Virtex7 VX485T & $100 \mathrm{MHZ}$ & 32 -bit Float & 61.62 & 0.22 & 1.33 & 3.31 \\
\hline$[106]$ & Zynq XC7Z020 & $100 \mathrm{MHz}$ & fixed & 12.73 & 0.6 & 5.48 & 7.27 \\
\hline$[36]$ (VGG) & StrtixV GSD8 & $120 \mathrm{MHz}$ & Fixed (8-16 bit) & 117.8 & 1.35 & 30 & 1.84 \\
\hline$[101]$ & Arria10 GX1150 & $385 \mathrm{MHz}$ & fixed & 1790 & 3.06 & 30.76 & 47.78 \\
\hline$[64]$ & Zynq XC7Z045 & $150 \mathrm{MHz}$ & fixed & 136.97 & 1.17 & 30.76 & 14.22 \\
\hline$[98]$ (ALexNet) & Virtex 7 485T (MLP) & $100 \mathrm{MHz}$ & 32 bit float & 85.2 & 0.35 & 30.94 & 11.21 \\
\hline$[98]$ (AlexNet) & Virtex 7 690T (MLP) & $100 \mathrm{MHz}$ & 32 bit float & 113.92 & 0.36 & 30.94 & 11.17 \\
\hline$[98]$ Squeezetet & Virtex 7 690T (MLP) & $170 \mathrm{MHz}$ & Fixed 16 bit & 909.7 & 1.53 & 30.94 & 126.34 \\
\hline$[107]$ (AlexNet) & Virtex7 VX485T & $100 \mathrm{MHz}$ & 32 bit float & 84.2 & 0.3 & 1.33 & $\mathrm{NA}$ \\
\hline$[108]$ (AlexNet) & Cyclone 5 & $100 \mathrm{MHz}$ & Fixed 16 bit & 12.11 & 1.39 & 1.33 & $\mathrm{NA}$ \\
\hline$[108]$ (AlexNet) & Virtex7 VX485T & $100 \mathrm{MHz}$ & Fixed 16 bit & 445 & 1.59 & 1.33 & $\mathrm{NA}$ \\
\hline$[106]$ & Zynq 7045 & $125 \mathrm{MHz}$ & Fixed 16 bit & 270 & $\mathrm{NA}$ & $\mathrm{NA}$ & 54 \\
\hline
\end{tabular}

Most of the mentioned works makes use of some of the common techniques like bit-width quantization and and efficient use of on-chip memory to tackle the resource requirements. Though, other techniques like use of systollic arrays, reduction of sparsity and usage of Winograd transform for MAC operations are mainly application specific and these techniques can be really helpful in implementing similar networks. Most of the FPGA based optimizations focuses more on improving the throughput than energy consumption. The performance analysis of the works mentioned in this section are mentioned in Table 7. Since all these works follow different benchmarks and different platforms, a fair comparison is not possible. The advantages of having a hardware framework for FPGA implementations are discussed in section 6 and more detailed comparison of FPGA implementation of CNNs is given in Tables 8, 9, and 10. 


\section{Hardware-aware Neural Architecture Search and hardware/software} co-design for FPGAs

A totally software-based approach does provide flexibility, but the sequential nature of execution would adversely affect the real-time execution and power consumption. Similarly, a totally hardware-based approach could be fast, but at the cost of reduced flexibility and accuracy. A hardware/software co-design offers a equilibrium between flexibility and speed while maintaining the accuracy and latency requirements. Some of the co-design approaches helps to reduce the parameter and network size. These approaches are discussed in detail in section 3 . Neural Architecture Search (NAS) as discussed in section 3.4 helps in automated generation of more accurate models, but are computationally expensive and hardware constraints put a restriction on deployment of NAS optimized models. A hardware/software co-design approach is required to address this issue and hardware-aware NAS approaches are becoming popular. The main idea is that the training is performed while taking the hardware constraints into consideration and testing is performed while taking DNN specifications into consideration. A co-design approach can target different hardware platforms to explore, and can generate solutions to satisfy the timing constraints while attaining a maximum accuracy. A few of such models with high impact targeting CNNs are discussed in this section.

MnasNet [23] is specifically targeted for mobile applications, and it does not require the model speed parameter like FLOPs for automation. The model itself analyzes the model speed by executing the model on a specific platform. By this way, the search can calculate the best trade-off for the particular mobile platform. MnasNet uses a factorized hierarchical search space to factorize the network into sequence of blocks and then a hierarchical search space is used to determine the layer architecture for each blocks. This model keeps each blocks to the same structure and hence the search space size is reduced multifold. In comparison, MnasNet achieves 1.5x speedup against MobileNetV2 [19] and 2.4x speedup against NASNet. In object detection using COCO dataset, this model outperforms mobilenet while at par in accuracy with SSD300 with 35x less computation cost.

AmoebaNet [24] uses the idea that the best architectures are evolved via mutating the existing ones in every iteration and these newest architectures are kept while the oldest architectures are removed. This approach is applied to NAS search space. The resulting architecture from NASNet formed by the Normal cells and Reduction cells will only have 2 hyper parameters and these parameters determines the tradeoff between accuracy and size. AmoebaNet achieves the best accuracy in CIFAR-10 dataset with $97.87 \%$ and a best accuracy $83.1 \%$ on ImageNet which is same as SENet [83] but SENet required more parameters and MAC operations to reach there as shown in Table 5. DARTS [25], discussed in section 3.4, also helps in reducing the search cost. Single-path NAS [109] treats the in-efficiency of NAS with another approach. It uses shared convolutional kernel parameters and a single path to encode all all architectural decisions compared to other differentiable NAS approches. This can significantly reduce the search cost and parameter count. The search cost is only 8 epochs (about 4 hours) while having an accuracy of $74.96 \%$.

$\mathrm{Wu}$ et. al introduced FBNet [110] which employs differential architecture search framework with gradient-based optimization. Instead of searching the whole 


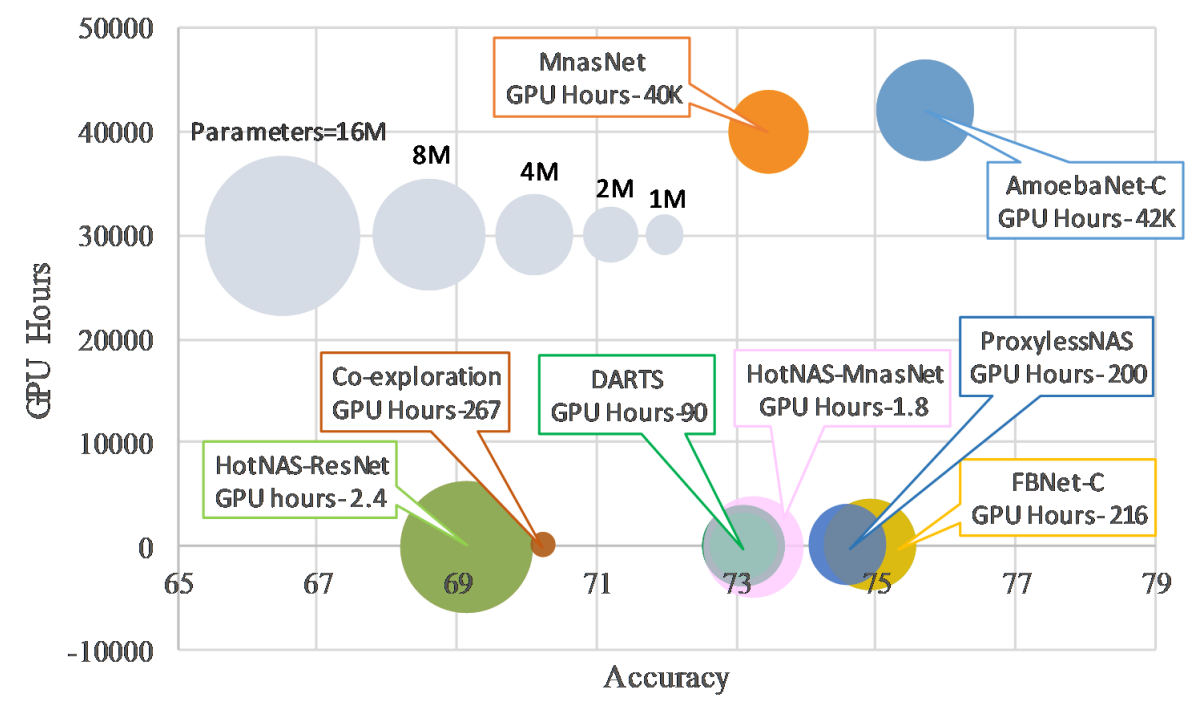

Fig. 6 NAS GPU Hours against number of parameters and accuracy

dataset, FBNet searches on a subset of the dataset. This technique reduces the seach cost, which is $420 \mathrm{x}$ less than MnasNet [23]. Also, FBNet can be upto $6.4 \%$ more accurate than MobileNet-v2. Whereas ProxylessNAS [111] learns directly on the large dataset on the target architecture than using a subset or proxy. ProxylessNAS shows 200x less training hours compared to MnasNet with better accuracy and lower GPU latency.

FNAS [28,112] introduces an FPGA-implementation aware NAS targeting resource constrained devices. Given an inference latency requirement, FNAS can generate an optimized model with guaranteed latency and maximum accuracy. It takes FPGA inference performance into consideration during network searching. FNAS employs a performance abstraction model to analyze the latency of neural architectures without training. This helps to quickly prune architectures that do not satisfy the specification and helps in achieving a higher efficiency. Jiang et. al [113] proposes HotNAS, a framework for hardware-aware neural architecture search which helps to reduce the search time. Within the $5 \mathrm{~ms}$ timing constraint, this work shows $5.79 \%$ gain in accuracy while having a $66 \%$ reduction in search time on Imagenet dataset when comparing with the existing models. EDD, a fully simultaneous, Efficient Differentiable DNN architecture and implementation co-search methodology is proposed in [114] which also helps in reducing the search time. Here, DNN search variables and Hardware varialbles are fused in the search space. The FPGA implementation shows 1.45\% higher throughput than DNNBuilder [115]. There are similar works such as NAIS [116] NASCaps [117], APNAS [118], and [119] which address this issues and explores the hardware-aware NAS techniques.

A well-optimized co-design approach is introduced by Hao et. al [120] which can generate board-level HLS design. This work proposes a simultaneous co-design flow, where the top-down approach focuses on a DNN-driven hardware 
design and the bottom-up approach focuses on hardware-aware DNN design. A hardware-aware DNN building block, Bundle-Arch is used to create DNN models, and a design search component Auto-DNN is used to explore DNN models taking the hardware constraints into consideration. Also, Tile-Arch and Auto-HLS is employed to generate and map DNNs to FPGAs. The results shows that this approach is more power efficient ( $40 \%$ less power) than the state-of-the-art implementations. SkyNet [121] proposes a hardware-efficient method for object detection and tracking employing the same bottom-up approach. A graphical representation of how the hardware aware NAS techniques reduced the search time and parameters compared to NAS (as shown in figure 2) is shown in figure 6. The chart includes only the networks targeting Imagenet dataset, and it can be seen that the GPU hours for search has been reduced to a bare 1.8 hours (HotNas) compared to 40,000 hours of MnasNet.

\section{Neural Network Libraries/Frameworks Targeted for FPGAs}

Neural network frameworks are helpful in reducing the workload of hardware accelerator designers by providing readily available building blocks. A number of frameworks/libraries are available for Neural Networks specifically targeting hardware. However, most of such frameworks concentrate on acceleration of training and performance and are not really suitable for hardware inference implementations. Frameworks such as TensorFLow[122], Keras[123], and Pytorch[124] provides optimized libraries for CNNs, but not much support for compilation on to hardware. This section shed light to some of the hardware frameworks targeting FPGAs, both high-level frameworks and ad-hoc model-to-FPGA compilers.

\subsection{High-level Frameworks}

High-level frameworks are written in high-level languages and can be mapped into hardware with the help of high level synthesis tools. Some of the high level frameworks targeting the hardware acceleration of CNNs are discussed in this section.

Caffe [125], developed by Berkeley Vision and Learning Center (BVLC) is a clean and modifiable framework for state-of-the-art deep learning algorithms, and a collection of reference models. The code is written in clean, efficient $\mathrm{C}++$, with CUDA used for GPU computation, and nearly complete, well-supported bindings to Python and MATLAB. OpenCL Caffe [126] is an approach to transform the Caffe framework into an OpenCL backend. Batching and multiple command queues are used in this for data and task parallelism. Cross platform portability is one of the main advantages of OpenCL Caffe, which can run in all devices which support OpenCL. Performance-wise, OpenCL Caffe batched manner can provide almost similar performance as Caffe with cuBLAS but lags behind significantly against Caffe with CuDNN. Still, in terms of performance/dollar OpenCL Caffe is competitive.

A Caffe based Framework model named Ristretto is presented in [66]. Along with other optimization methods like dynamic and mixed fixed point, Ristretto 
takes a trained model as input and compresses the model to even smaller size. More specific details about the optimization techniques used in Ristretto is explained in section 3.2. FixCaffe [127] Framework is also based on the Caffe [125] model. This model modifies the Caffe model by substituting the floating point matrix multiplications with low precision fixed point matrix multiplications. To improve the efficiency, stochastic rounding scheme is used. Compared to the floating point version, the 8-bit implementation of LeNet- 5 shows only $0.3 \%$ loss in accuracy for 1000 iterations. While, 10-bit precision model gives almost no loss in accuracy. The memory footprint also reduced to only $31.25 \%$ and $25 \%$ respectively for 10 -bit precision and 8-bit precision.

Theano [128], a powerful python based framework for numerical computations, take computations expressed in NumPy's [129] syntax and convert it into $\mathrm{C}++$ or CUDA and then compiles it into dynamically loaded python modules. Implementation of machine learning algorithms in Theano for CPU are 1.6x to 7.5x faster than other programming alternatives like $\mathrm{C} / \mathrm{C}++$, Numpy or Matlab. Meanwhile GPU implementation can be $6.5 \mathrm{x}$ to $44 \mathrm{x}$ faster.

TensorRT [130] from nvidia is a high performance deep learning library which can deliver maximum inference throughput and efficiency. TensorRT accelerates GPU inference by optimizing trained NNs for run-time performance. Tensorflow Lite Micro [131] is introduced recently for machine learing inference applications on embedded systems. Similar to Tensorflow Lite Micro, a number of frameworks targeting embedded devices are available such as TinyEngine [132], Embedded Learning Library [133], uTensor [134], Glow [135], and STM32Cube.AI [136].

MXNet or mix-net [137] is a special multi-language machine learning library for various heterogeneous systems ranging from embedded devices to distributed GPU clusters. MXNet embeds multiple host language like $\mathrm{C}++$, Python, $\mathrm{R}$, Go, and Julia. While comparing with other popular frameworks, MXNet give similar performance as Caffe and Torch7 against GoogLeNet, AlexNet, and VGG, and it is twice as fast as Tensorflow.

\subsection{Model-to-FPGA compilers}

Model-to-FPGA compilers are hardware design automation frameworks which can generate HLS or RTL codes based on the network parameters. A few of such frameworks along with some hybrid (HLS-RTL) approaches are discussed in this section.

\subsubsection{HLS based approaches}

Caffeine [138] is a hardware/software co-designed library for FPGA hardware accelerators for CNN. Unlike the other frameworks/libraries for FPGA implementation, Caffeine is implemented using high level synthesis and hence portable across multiple platforms. Caffeine design is focused more on the bandwidth optimization by the memory access reorganization. Another feature of Caffeine is that instead of using the conventional matrix multiplication, it uses a uniformed convolutional matrix multiplication. This gives Caffeine fully connected layers a large speedup when compared to previous works. This paper also provides the results of integration of the Caffe framework with Caffeine. Ceva Deep Neural 
Network (CDNN2) Framework [139] can also accept either Caffe or TensorFlow representations and can generate a hardcore or soft-core representations for SoC FPGAs.

fpgaConvNet [106] is a neural network framework for implementing CNNs on FPGA. The workflow of the model is in such a way that a Synchronous Data flow (SDF) model is generated by analyzing the ConvNet workload and FPGA resource constraints. The computing system is modelled as a directed SDF graph. Then, based on the application level performance requirements, the design space exploration is formed as a mathematical optimization problem with respect to the throughput, latency and other use defined requirements. Finally, an optimized streaming accelerators is generated. fpgaConvNet gives fairly accurate performance predictions and achieves improvements in performance density and performance efficiency over existing FPGA and embedded GPU models.

A Framework targeted for fast and flexible FPGA accelerators is FINN [140], based on binarized neural networks [71],[61]. Since FPGAs are best known for giving peak performance in binary operations than floating point operations, this framework based on BNNs can improve the performance on FPGAs. FINN uses a heterogeneous streaming architecture and because of the compact size of binarized CNN, all parameters can be stored in the on-chip memory. The design flow of FINN is based mainly on a FINN synthesizer. A user supplied FPS target along with a Theano trained BNN is inputted to the Synthesizer. The synthesizer analyzes the network in order to meet the target specifications and determines optimized folding parameters. By using the parameters, a synthesizable $\mathrm{C}++$ description of a heterogeneous streaming architecture is generated. With the help of a customized FINN hardware library, Vivado generates a hardware description. Comparing with other works, we can say that FINN doesn't really improve the accuracy significantly, but this 1-bit precision network outperforms a 32 bit model by thousands fold in terms of speed.

Angel-Eye [141] is a programmable and flexible CNN accelerator architecture which can compile CNNs to FPGAs. Angel-Eye makes use of algorithmic optimizations such as quantization and it has a custom instruction set for the hardware traslation. It employs a single software-programmable computation engine, which is composed of arrays of processing elements (PE). PEs consists of convlution blocks, adder tree and pooling path and each individual PEs have access to input feature maps in a parallel manner. Each PE can generate feature maps with individual set of kernels. The compiler translates the CNN to executable instruction sequences for the hardware. This model is CNN-independant and each CNNs will be mapped with different instruction sequences. Angle-Eye delivers $6 \mathrm{x}$ power-efficiency and 5x speed compared to other FPGA implementation on the same platform.

CascadeCNN [142] is a very different and efficient approach of an automated toolflow for improving the performance, mainly the accuracy, of precision-quantized CNN models by exploiting the computation time-accuracy trade-off. Similar to some other models discussed, CascadeCNN framework accepts a Caffe network description file along with the target hardware resource details, a user defined error tolerance metric and an evaluation set and generates a highest performing configuration of the architecture by analyzing the design space by means of a roofline model. The system outputs a two-stage hardware architecture which consists of a low precision unit (LPU), high precision unit (HPU) and a 
confidence evaluation unit (CEU). LPU uses low precision arithmetic to provide high throughput whereas HPU provides the same accuracy as the reference model. CEU evaluates the misclassified samples by the LPU and forward to HPU for re-processing. The advantage of this model is that the HPU performs computation based on the CEU analysis and most of the computation is performed by LPU, which improves the throughput. Also, the low precision of the output is restored with respect to user's requirements. To evaluate the model, two FPGAs Xilinx Zynq ZC706 and UltraScale+ ZCU102 are used and on both the platforms implementation of pretrained models AlexNet and VGG-16 showed a $48 \%$ and $55 \%$ improvement compared to baseline models. Another efficient design space analysis approach is explored in [143]. This work makes use of uniform templates based on Winograd algorithm to generate hardware accelerator architectures for acceleration of $2 \mathrm{D}$ and $3 \mathrm{D}$ CNNs.

Design and development of FPGA based hardware accelerator framework for Deep convolutional Neural Networks (DCNN) with a systematic approach is discussed in [107]. The proposed architectural template of this model exploits all sources of parallelism in a given DCNN algorithm. This work also develops a design space exploration algorithm to design the most efficient architecture based on the target platform. The architecture is designed in 4 stages in such a way that it can exploit inter and intra layer parallelism, inter and intra output parallelism, and inter and intra kernel parallelism. A CNN implementation framework which proposes an analytical model along with an automated design space exploration methodology to optimize CNN implementation on FPGAs using stacked systolic arrays is discussed in paper [33]. A Comparison of [107] and [33] with some of the other models is given in Table 7 .

\subsubsection{RTL based approaches}

DeepBurning [37] is a framework which focuses on automated hardware optimization for neural networks specifically on FPGAs. DeepBurning framework takes the Caffe-script as input and according to the best hardware configuration and resource constraints, it generates RTL code for the accelerator with DeepBurning RTL generator and a dedicated IP library. Software-hardware co design is performed by integrated compiler which is essential in folding the network within the user constraints. Also, DeepBurning framework is able to effectively accelerate neural network models by harnessing the data locality and memory access pattern in neural network models. The generated DeepBurning implementation models shows greater power efficiency compared to the other competing implementations. HeteroCL [144] also provides a similar framework based on HLS, composed of a Python-based domain-specific language (DSL) and an FPGA-targeted compilation flow.

CaFPGA [145] is Framework for automatic generation of CNN reconfigurable accelerator on FPGA, which is very much similar to DeepBurning [37]. The model presents an auto-design framework which works in such a way that it takes the Caffe description text as its input and then with the help of the proposed IP library, the Caffe script is translated to a Hardware Design Flow Graph (HDFG), similar to SDFG in [106]. The proposed Design Space Exploration algorithm then takes the HDFG, IP Library and Resource constraints as the inputs, and outputs an optimized HDFG from which the hardware code is generated. This model digs 
deep into the design space exploration to find the best accelerator design as per user requirements based on the design and resource constraints for specific CNN model and the FPGA platform. It makes use of both folding and pipeline structures of CNN by combining these two structures to create a layer-folding pipeline structure. This combined structure can somewhat eliminate the disadvantages of both these standalone structures and hence improves the efficiency of the model.

DNNWeaver [146] automates the process of accelerator generation for the selected DNN and FPGA pair using hand-optimized design templates in Verilog. It uses the Caffe description model as input. The automated workflow of DNNWeaver consists of a translator-which converts the caffe specifications to a macro data flow graph in the form of a coarse-grained ISA, a design weaver-which converts the macro data flow graph instructions to a synthesizable Verilog implementation of the accelerator code, and an integrator-which adds the memory interface code to the generated accelerator. The framework mainly targeted on ZynQ and Stratix FPGAs and provides considerable speedup against CPUs. Against Xeon E3, implementation of Cifar-10 and MNIST gives a maximum speedup of $11.6 \mathrm{x}$ and $7.9 \mathrm{x}$ respectively for Stratix while a maximum speedup of $1.8 \mathrm{x}$ and $1.4 \mathrm{x}$ respectively for ZynQ. Similarly, the performance per watt against the same implementations gives a higher performance of $33 \mathrm{x}$ and $22 \mathrm{x}$ respectively for Stratix and 52x and 41x respectively for ZynQ. In comparison with GPUs, the model gives a speed decline, but shows better power efficiency than GPUs.

PLACID [108] is a hardware generator platform which takes DCNN description as input and then generate an optimized hardware description for FPGAs in Verilog. The proposed architecture claims to utilize the different sources of parallelism in DCNN algorithms. Also, the unique properties of FPGAs are exploited to develop a memory hierarchy that reduces the off-chip bandwidth. For memory optimization, techniques like tiling and Input/output feature map reuse, etc. are used. The architecture support two types of data access approaches namely non-critical memory access and critical memory access for data access optimization. Another main feature is the Fat Convolution Engine (FCE), which is the smallest processing unit which can intra-kernel parallelism by parallelizing each convolution operation. A parallel convolution engine (PCE) consists of multiple FCEs in parallel. This model uses 16-bit fixed point format.

Although most of the the model-to-FPGA compilers focus on performance than resource utilization, DNNBuilder [115] is an end-to-end automation tool which can generate higly optimized RTL code for FPGAs. DNNbuilder aims to target high-performance while addressing the limited resource constraints. This work employs algorithmic optimizations such as quantization and architectural optimizations such as fine-grained layer-based pipeline architecture and a column-based cache scheme. An automatic design space exploration tool helps in efficient resource allocation. It shows $43 x$ reduction in BRAM and $7.7 x$ reduction in latency while achieving $5.15 x$ faster performance comparing with the best available FPGA implementation. A similar automated RTL code generator for FPGA and ASIC platforms is AutoDNNchip [147]. Such a platform can significantly reduce the chip design time. AutoDNNchip accepts the specifications from the user and with the help of two integrated enablers Chip Predictor and Chip Builder, it can automatically generate optimized designs targeting FPGA/ASIC. Another work on automated RTL code generation is done in [148]. This work concenrates on both algorithmic and hardware optimizations along with a tool to generate verilog code 
for FPGA. With the help of a proposed Concatenate-and-Pad (CaP) technique, wasted computations in Overlap and Add (OaA) technique for frequency domain convolution is eliminated. The evaluation results also shows better throughput with moderately less resource utilization compared to other works as shown in Table 8 .

Table 8 Throughput comparison of some of the frameworks on FPGA

\begin{tabular}{|c|c|c|c|c|c|c|c|c|}
\hline Model & FPGA & $\begin{array}{l}\text { Design } \\
\text { Entry }\end{array}$ & $\begin{array}{c}\text { Frequency } \\
(\mathrm{MHz})\end{array}$ & $\begin{array}{c}\text { CNN size } \\
(\mathrm{GOP})\end{array}$ & $\begin{array}{l}\text { Weights } \\
\text { (MB) }\end{array}$ & Precision & $\begin{array}{c}\text { Throughput } \\
\text { (GOPs) }\end{array}$ & $\begin{array}{c}\text { Performance } \\
\text { Density } \\
\text { (GOPs/DSP } \\
\text { cycle) }\end{array}$ \\
\hline [149] (NIN) & Stratix-V GXA7 & RTL & 100 & 2.2 & 7.59 & Fixed 8-16 bit & 117.3 & 4.58 \\
\hline [150] (NIN) & Stratix-V GXA7 & RTL & 150 & 2.2 & 7.59 & Fixed 16 bit & 282.67 & 7.36 \\
\hline [145] CaFPGA (AlexNet) & Virtex-7 VX690T & RTL & 137 & 1.46 & 60.95 & Fixed 8-16 bit & 593.5 & 1.52 \\
\hline [145] CaFPGA (VGG16) & Virtex-7 VX690T & RTL & 150 & 30.94 & 138.36 & Fixed 8-16 bit & 638.9 & 1.43 \\
\hline [138] Caffeine (VGG16) & Virtex-7 VX690T & HLS & 150 & 30.94 & 276.72 & Fixed 16 bit & 354 & 0.83 \\
\hline [29] (AlexNet) & Virtex-7 VX485T & $\mathrm{C}$ & 100 & 1.33 & $2.33 \mathrm{M}$ & Float 32 bit & 61.6 & 0.22 \\
\hline [36] (AlexNet) & Stratix-V GXA7 & OpenCL & 193.6 & 1.46 & 60.95 & Fixed 8-16 bit & 60.2 & 1.21 \\
\hline [36] (AlexNet) & Stratix-V GXA7 & RTL & 100 & 1.46 & 60.95 & Fixed 8-16 bit & 114.5 & 4.47 \\
\hline$[148]$ (AlexNet) & Stratix-V & RTL & 200 & 1.46 & 60.95 & Fixed 16 bit & 780.6 & 15.24 \\
\hline [151] FP-DNN (VGG19) & Stratix-V GSMD5 & HLS-RTL & 150 & 30.95 & $\mathrm{NA}$ & Fixed 16 bit & 364.4 & 2.34 \\
\hline [150] (VGG16) & Stratix-V GXA7 & RTL & 150 & 30.95 & 138.3 & Fixed 16 bit & 352.24 & 9.17 \\
\hline [33] (VGG16) & Arria $10 \mathrm{GX} 1150$ & RTL & 231.85 & 30.94 & 138.36 & Fixed 8-16 bit & 1171.3 & 3.37 \\
\hline$[150]$ (VGG16) & Arria 10 GX1150 & RTL & 200 & 30.95 & 138.36 & Fixed 16 bit & 720.15 & 2.37 \\
\hline$[64]$ (VGG16) & ZynQ XC7Z045 & RTL & 150 & 30.76 & 50.18 & Fixed 16 bit & 136.97 & 1.17 \\
\hline$[148]$ (VGG16) & Stratix-V & RTL & 200 & 30.95 & 138.36 & Fixed 16 bit & 669.1 & 13.07 \\
\hline
\end{tabular}

\subsubsection{Hybrid approaches}

The inefficiency of high level synthesis (HLS) despite having the flexibility and a faster design time is pointed out in ALAMO by Ma et. al $[152,149,150]$, and comes up with a modular and scalable hardware design framework which integrates both HLS and RTL design to optimize the CNN implementation on FPGA. The core of the framework is a CNN RTL compiler which converts Caffe or Theano generated CNN models to generate parameterized CNN RTL modules by also considering the degree of parallelism set by the users. The FPGA implementation on Altera FPGA makes use of Nios II softcore processor for memory transfer from flash to DDR3. One drawback which can pull back the performance of this model is that the weights are stored in external RAM, which can considerably slow down the execution and increase the power consumption. A similar hybrid approach is presented in FP-DNN [151]. FP-DNN make use of optimized RTL-HLS hybrid templates to automatically generate hardware implementation from Tensorflow-described CNNs. FP-DNN divides the operations in to computation-intensive part and layer-specific part. The computation-intensive part employs a high-performance matrix multiplication kernel to accelerate the inference, whereas the layer-specific part is composed of an optimized communication bandwidth.

The above mentioned libraries/frameworks mitigate the hardwork required by the hardware designer. However, an automated approach on frameworks to generate hardware code for FPGAs are still not at its best. Most of the frameworks provides better design space exploration to generate a suitable architecture, but translation of this architecture into FPGA still takes some effort. A comparison of CNN implementation of hardware architectures based on some of the discussed works are given in tables 8, 9 and 10. Since different models use different designs, a 
fair comparison is not feasible. Mostly the comparison is done on the works which uses ImageNet dataset for evaluation.

Table 9 Convolution layer latency and throughput

\begin{tabular}{|l|l|l|c|c|c|}
\hline Model & FPGA & $\begin{array}{c}\text { Design } \\
\text { Entry }\end{array}$ & $\begin{array}{c}\text { Number of } \\
\text { convolution } \\
\text { layers }\end{array}$ & $\begin{array}{c}\text { Convolution } \\
\text { time (ms) }\end{array}$ & $\begin{array}{c}\text { Convolution } \\
\text { throughput } \\
\text { (GOPS) }\end{array}$ \\
\hline$[29]$ (AlexNet) & Virtex-7 VX485T & HLS & 5 & 21.61 & 61.6 \\
\hline$[36]$ (AlexNet) & Stratix-V GXA7 & HLS & 5 & 19.86 & 67.5 \\
\hline$[145]$ CaFPGA (AlexNet) & Virtex-7 VX690T & RTL & 5 & 4.92 & NA \\
\hline$[138]$ Caffeine (AlexNet) & UltraScale KU060 & HLS & 5 & NA & 163 \\
\hline$[138]$ Caffeine (VGG16) & UltraScale KU060 & HLS & 13 & NA & 310 \\
\hline$[106]$ fpgaConvNet (AlexNet) & Zynq XC7Z045 & HLS & 5 & 8.22 & 197.4 \\
\hline$[106]$ fpgaConvNet (VGG16) & Zynq XC7Z045 & HLS & 13 & 249.5 & 155.81 \\
\hline$[146]$ DNNWeaver (AlexNet) & Zynq XC7Z020 & HLS & 5 & NA & 20.16 \\
\hline$[146]$ DNNWeaver (VGG16) & Zynq XC7Z020 & HLS & 13 & NA & 31.35 \\
\hline$[153]$ Snowflake (AlexNet) & Zynq XC7Z045 & HLS & 5 & 9.95 & 120.3 \\
\hline$[37]$ DeepBurning (AlexNet) & Zynq XC7Z045 & RTL & 5 & 12.3 & 108.25 \\
\hline$[152]$ ALAMO (AlexNet) & Stratix V GXA7 & RTL & 13 & 9.92 & 134.1 \\
\hline$[149]$ ALAMO (NIN) & Stratix-V GXA7 & RTL & 12 & 18.75 & 117.3 \\
\hline$[150]$ ALAMO(VGG-16) & Arria 10 GX1150 & RTL & 13 & 27.23 & NA \\
\hline$[64]$ (VGG-16) & Zynq XC7Z045 & RTL & 13 & 163.42 & 187.8 \\
\hline$[33]$ (VGG-16) & Arria 10 GX1150 & RTL & 13 & 26.85 & NA \\
\hline$[141]$ Angel-Eye (VGG16) & Zynq XC7Z045 & HLS & 13 & 163.42 & 187.8 \\
\hline$[145]$ CaFPGA(VGG-16) & Virtex-7 VX690T & RTL & 13 & 48.43 & NA \\
\hline$[94]$ NullHop(VGG-16) & Zynq 7100 & RTL & 13 & 72.94 & 420.83 \\
\hline
\end{tabular}

NA-Not Available

Table 10 FPGA Resource utilization

\begin{tabular}{|l|l|c|c|c|c|c|c|}
\hline Model & FPGA & $\begin{array}{c}\text { Design } \\
\text { Entry }\end{array}$ & $\begin{array}{c}\text { Frequency } \\
(\mathrm{MHz})\end{array}$ & $\begin{array}{c}\text { LUT/ALM } \\
\text { utilization }\end{array}$ & $\begin{array}{c}\text { DSP } \\
\text { utilization }\end{array}$ & $\begin{array}{c}\text { On-chip } \\
\text { RAM utilization }\end{array}$ & $\begin{array}{c}\text { Total memory } \\
\text { utilized (MB) }\end{array}$ \\
\hline$[29]$ (AlexNet) & Virtex-7 VX485T & C & 100 & $186251(61.3 \%)$ & $2240(80 \%)$ & $1024(50 \%)$ & 36.86 \\
\hline$[64]$ (VGG-16) & Zynq XC7Z045 & RTL & 150 & $182616(83.5 \%)$ & $780(89.2 \%)$ & $486(86.7 \%)$ & 17.49 \\
\hline$[36]$ (AlexNet) & Stratix-V GXA7 & OpenCL & 193.6 & $\sim 114000(49 \%)$ & $256(100 \%)$ & $1893(74 \%)$ & 37.86 \\
\hline$[149]$ (AlexNet) & Stratix-V GXA7 & RTL & 100 & $\sim 121000(52 \%)$ & $256(100 \%)$ & $1552(61 \%)$ & 31.04 \\
\hline$[19]$ (NIN) & Stratix-V GXA7 & RTL & 100 & $\sim 112000(48 \%)$ & $256(100 \%)$ & $2330(91 \%)$ & 46.6 \\
\hline$[150]$ (NIN) & Stratix-V GXA7 & RTL & 150 & $\sim 256400(96 \%)$ & $256(100 \%)$ & $1510(59 \%)$ & 30.2 \\
\hline$[150]$ (VGG-16) & Stratix-V GXA7 & RTL & 150 & $\sim 212100(90 \%)$ & $256(100 \%)$ & $2202(86 \%)$ & 44.04 \\
\hline$[138]$ (VGG-16) & Virtex-7 VX690T & HLS & 150 & $\sim 561000(81 \%)$ & $2833(79 \%)$ & $1248(42 \%)$ & 44.92 \\
\hline$[3]$ (VGG-16) & Arria 10 GT1150 & RTL & 231.85 & $\sim 313000(73 \%)$ & $1500(49 \%)$ & $1668(61 \%)$ & 33.36 \\
\hline$[150]$ (VGG-16) & Arria 10 GX1150 & RTL & 200 & $\sim 131700(30 \%)$ & $1518(100 \%)$ & $2225(82 \%)$ & 44.5 \\
\hline$[151]$ FP-DNN & Stratix-V GSMD5 & HLS-RTL & 150 & $42349(25 \%)$ & $1036(65 \%)$ & $919(46 \%)$ & 18.38 \\
\hline$[145]$ CaFPGAA(AlexNet) & Virtex-7 VX690T & RTL & 137 & $317846(73 \%)$ & $2843(80 \%)$ & $925(63 \%)$ & 33.3 \\
\hline$[15]$ CaFPGA(VGG-16) & Virtex-7 VX690T & RTL & 150 & $268152(62 \%)$ & $2967(82 \%)$ & $1247(84.8 \%)$ & 44.89 \\
\hline$[94]$ NullHop(VGG-16) & Zynq 7100 & RTL & 60 & $\sim 227000(83 \%)$ & $128(6.3 \%)$ & $386(51.1 \%)$ & 13.89 \\
\hline
\end{tabular}

\subsection{Hardware Description Language Platforms}

In this survey, we concentrate mainly on FPGA based implementation platforms. In FPGA based system design, even though RTL based hardware designs using Verilog and VHDL are considered to be more flexible and efficient way of designing, the design complexities and time-to-market are limiting this approach. One way to reduce this complexities and reduce development time is to employ High Level Synthesis (HLS) tools. HLS tools use high level programming languages or Graphical modelling like MATLAB Simulink to generate RTL level abstraction 
for the targeted FPGA device. Similarly in the case of GPUs, high level language based libraries like CUDA and more optimized high level languages like OpenCL are available. SystemVerilog is another language which has the capabilities of both RTL and high level language and is more flexible than RTL alone. The work in [32] makes use of SystemVerilog in the implementation of an FPGA based CNN accelerator. There are a number of tools and platforms which support FPGAs and a well detailed list is given in [154]. Vivado is one of the most used HLS tool from Xilinx which supports $\mathrm{C} / \mathrm{C}++/$ OpenCL and another widely used tool is Quartus Prime from Intel. Xilinx Vitis is a unified software platform with optimized hardware libraries for AI. With the popularity of OpenCL, OpenCL specific tools like Intel SDK and SDAccel were introduced. Compared to other high level languages, OpenCL and Python based design are getting more popular because of the presence of more optimized libraries and the ability to exploit the parallelism. Compilers/libraries like JHDL and RHDL are also available which can convert Java and Ruby into RTL code.

Frameworks like Caffeine [138] and FINN [140] are totally implemented using HLS with high level of optimization for CNNs. The article in [155] gives an insight about using OpenCL for HLS using Vivado. Even though Vivado supports OpenCL, it is unable to exploit all the capabilities of OpenCL. An HLS based OpenCL design methodology for HPC systems is explored in [156] and it presents an efficient FPGA implementation of KNN algorithm, Monte Carlo methods for financial models, and Bitonic sorting algorithm using OpenCL on Xilinx SDAccel tool. The results were very impressive as it showed a $28000 \mathrm{x}$ improvement in energy efficiency with the best case FPGA when compared to the best case GPU for KNN algorithm and 769x improvement in speed. The FPGA implementation outperformed the GPU on the implementation of Monte Carlo models also. OpenCL based implementation on Altera FPGAs is proposed in [101] and [99]. The detailed design methodology and results are discussed in section 4.2.2.

A python based HLS compiler is discussed in [157], which can interpret a python based algorithmic description to a synthesizable VHDL code. This work was followed by another version of python based compiler named RPython [158] which can generate implementations for dynamic languages. MyHDL [159] is another python based HDL, which is a package in python which uses Python constructs to support hardware description, modelling, verification and conversion. Similar to MyHDL, there are approaches like PyMTL [160], PyHDL [161], PyRTL [162] and PHDL [163] to use Python as an HDL. One important thing to mention here is that all these python based HDLs do not take a high level program as an input, but it helps the user to use the benefits of a high level language to describe a hardware level abstraction, which makes things easier for the programmer.

\section{Analysis and Future Directions}

The challenges in the inference implementation of CNNs are on FPGAs are mainly bounded by computation overhead, on-chip memory utilization, and accuracy. Computation overhead and memory bandwidth affects the energy use and latency. All these considerations are not mutually exclusive. There are always trade-offs among throughput, latency, resource utilization, power consumption and accuracy. 
Design for accuracy increases the network size and hence increases resource utilization, latency, and power consumption. All these factors need to be considered while designing for hardware and the design should be optimized for the particular metric of greater importance. Algorithmic optimization also plays an important role to a great extent in efficient implementation of CNNs on resource-constrained devices. However, most of the algorithmic optimizations bring great challenges when FPGA implementation is considered.

Accuracy is one of the major challenges in hardware design. The optimizations to improve the accuracy often increase the size of the network resulting in computation overhead as shown in Table 5 and figure 2, which limits the design space of hardware implementations. Throughput and latency are correlated and optimizing for these metrics can adversely affect the resource utilization and power consumption. For automated NAS networks, even though accuracy is high, the computation overhead and size of the network for training and inference is very high. We can see from figure 2 that as the accuracy increases, the parameter count and MAC operations increase drastically. This trade-off cannot be avoided while considering the hardware implementation. Data-quantization techniques somewhat help to reduce the computational requirements and also reduces the memory requirements, but this results in reduced accuracy. Networks like Binarized Neural Networks [69] can also provide solutions to reduce resources in hardware implementation. Network size is another major issue. The optimizations in algorithms help to reduce the size of the network and hence the memory footprint. As seen from deep compression [47], network pruning followed by quantization and huffman coding reduces the network size further. Furthermore, improved dataflow modeling are required to overcome the issues related to memory access and to improve data reuse.

To tackle these challenges in the future, numerous approaches are being put forward. The complexities of GPUs as accelerators are eventually making a move towards FPGAs. Nevertheless, the translation of the algorithmic optimizations on hardware is not easy. Considering the trade-offs like network size and accuracy on algorithmic level, optimizing for accuracy with the reduction of resources at the same time is a cumbersome task. Also, the optimizations on hardware is very much limited compared to the range of optimizations we can perform in software implementations. Even though hardware description languages like verilog and VHDL provides flexibility in design, it is tiresome for a hardware designer to use HDL for implementing present day CNNs. HLS based tools help to reduce both design time and complexity. Architectural optimizations alone is not going to help hardware designers to implement deep learning algorithms on FPGAs. Hence more focus should be given to developing suitable HLS based tools which can help in efficient inference acceleration. A HLS-RTL hybrid approach $[152,151]$ would also be very much beneficial as shown by prior works.

Considering the optimization techniques targeted on hardware, the availability of open source high-level frameworks help CPU/GPU implementations to a great extent in terms of design time and efficient compilation of complex algorithms while transferring to hardware. A number of frameworks are there to support GPUs and GPU programmers who make use of such frameworks don't have to worry about translation of back-propagation, or low-level implementation on to the hardware. Though high-level frameworks such as Caffe, Theano, MXNet, and FINN help to train the network for hardware implementation, compiling 
the networks onto hardware is still a manual task. Model to FPGA Frameworks discussed in section 6.2 are helpful in generating the hardware designs based on algorithmic optimizations. However, the efficiency of hardware libraries used in such frameworks is also limited while compiling for resource constrained devices. Frameworks which support FPGA-based CNN training while providing support for integrating the existing frameworks in the hardware design must be given priority. An automated approach to generate hardware design as per the user specifications from pre-built hardware libraries would be very much useful for FPGA-based accelerator design. Such high level frameworks with well-optimized libraries can transfer the algorithmic optimizations to hardware efficiently compared to manual compilation by the designer.

Hardware-aware NAS approaches are very much promising for hardware implementations. A co-design approach where including the hardware parameters during training and optimization helps in a more accurate, resource-efficient, power-efficient hardware architectures. In future, there will be a lot dependence on such hardware-aware NAS frameworks for developing high-performing architectures on hardware. Another important area to be mentioned is evolutionary computations. Merchant [164] has shown that it is possible to change the network at run-time with the help of genetic algorithms. NAS architectures such as DARTS [25] have shown the positive impact of evolutionary computations in the architecture optimization. As unsupervised learning becoming an important future direction of CNNs, addressing unlabelled data at run-time would be challenging. Reconfigurable hardware with the help of evolutionary computations could be a step to address these challenges in future.

\section{Conclusion}

In this paper, a thorough study of the available techniques for optimization of convolutional neural networks both on algorithmic and hardware level is presented. It can be concluded from the study that hardware design approaches are very much influenced by both algorithmic and architectural optimizations. Though, the feasibility of translating algorithmic optimizations into hardware is still difficult. The need of the hour for FPGA based implementations is high-level frameworks which can integrate and compile the algorithmic optimization techniques to hardware. It is worth mentioning that hardware design approaches are lagging behind the advances in algorithms and hence more attention must be given to figure out the efficient hardware implementation approaches making use of evolutionary computations and hardware-software co-design approaches.

\section{References}

1. A. Putnam and et al. A reconfigurable fabric for accelerating large-scale datacenter services. IEEE Micro, 35(3):10-22, 2015.

2. Microsoft Research Blog. Microsoft unveils Project Brainwave for real-time AI. https://www.microsoft.com/en-us/research/blog/microsoft-unveils-project-brainwave, 2017.

3. F. Rosenblatt. The perceptron: A probabilistic model for information storage and organization in the brain. Psychological Review, pages 65-386, 1958. 
4. Warren Mcculloch and Walter Pitts. A logical calculus of ideas immanent in nervous activity. Bulletin of Mathematical Biophysics, 5:127-147, 1943.

5. H. Ackley, E. Hinton, and J. Sejnowski. A learning algorithm for boltzmann machines. Cognitive Science, pages 147-169, 1985.

6. Y. Lecun, L. Bottou, Y. Bengio, and P. Haffner. Gradient-based learning applied to document recognition. In Proceedings of the IEEE, pages 2278-2324, 1998.

7. Alex Krizhevsky, Ilya Sutskever, and Geoffrey E. Hinton. Imagenet classification with deep convolutional neural networks. In Advances in Neural Information Processing Systems, page 1097-1105, 2012.

8. Min Lin, Qiang Chen, and Shuicheng Yan. Network in network. arxiv:1312.4400, 2014.

9. Christian Szegedy, W. Liu, Y. Jia, Pierre Sermanet, Scott Reed, Dragomir Anguelov, D. Erhan, V. Vanhoucke, and Andrew Rabinovich. Going deeper with convolutions. 2015 IEEE Conference on Computer Vision and Pattern Recognition (CVPR), pages $1-9,2015$.

10. Karen Simonyan and Andrew Zisserman. Very deep convolutional networks for large-scale image recognition, 2015.

11. Kaiming He, Xiangyu Zhang, Shaoqing Ren, and Jian Sun. Deep residual learning for image recognition. In Proceedings of the IEEE Conference on Computer Vision and Pattern Recognition (CVPR), June 2016.

12. Jia Deng, W. Dong, R. Socher, L. Li, K. Li, and Li Fei-Fei. Imagenet: A large-scale hierarchical image database. In CVPR 2009, 2009.

13. Forrest N. Iandola, Matthew W. Moskewicz, Khalid Ashraf, Song Han, William J. Dally, and Kurt Keutzer. Squeezenet: Alexnet-level accuracy with 50x fewer parameters and $¡ 1 \mathrm{mb}$ model size. ICLR, 2017.

14. J. Redmon, S. Divvala, R. Girshick, and A. Farhadi. You only look once: Unified, real-time object detection. In 2016 IEEE Conference on Computer Vision and Pattern Recognition $(C V P R)$, pages $779-788,2016$.

15. Andrew G. Howard, Menglong Zhu, Bo Chen, Dmitry Kalenichenko, Weijun Wang, Tobias Weyand, Marco Andreetto, and Hartwig Adam. Mobilenets: Efficient convolutional neural networks for mobile vision applications. CoRR, 2017.

16. G. Huang, Z. Liu, L. Van Der Maaten, and K. Q. Weinberger. Densely connected convolutional networks. In 2017 IEEE Conference on Computer Vision and Pattern Recognition (CVPR), pages 2261-2269, 2017.

17. Xiangyu Zhang, Xinyu Zhou, Mengxiao Lin, and Jian Sun. Shufflenet: An extremely efficient convolutional neural network for mobile devices. CoRR, abs/1707.01083, 2017.

18. A. Howard, M. Sandler, B. Chen, W. Wang, L. Chen, M. Tan, G. Chu, V. Vasudevan, Y. Zhu, R. Pang, H. Adam, and Q. Le. Searching for mobilenetv3. In 2019 IEEE/CVF International Conference on Computer Vision (ICCV), pages 1314-1324, 2019.

19. M. Sandler, A. Howard, M. Zhu, A. Zhmoginov, and L. Chen. Mobilenetv2: Inverted residuals and linear bottlenecks. In 2018 IEEE/CVF Conference on Computer Vision and Pattern Recognition, pages 4510-4520, 2018.

20. Ningning Ma, Xiangyu Zhang, Hai-Tao Zheng, and Jian Sun. Shufflenet v2: Practical guidelines for efficient cnn architecture design. In Proceedings of the European Conference on Computer Vision (ECCV), September 2018.

21. B. Zoph, V. Vasudevan, J. Shlens, and Q. V. Le. Learning transferable architectures for scalable image recognition. In 2018 IEEE/CVF Conference on Computer Vision and Pattern Recognition, pages 8697-8710, 2018.

22. Chenxi Liu, Barret Zoph, Jonathon Shlens, Wei Hua, Li-Jia Li, Li Fei-Fei, Alan L. Yuille, Jonathan Huang, and Kevin Murphy. Progressive neural architecture search. CoRR, abs/1712.00559, 2017 .

23. Mingxing Tan, Bo Chen, Ruoming Pang, Vijay Vasudevan, and Quoc V. Le. Mnasnet: Platform-aware neural architecture search for mobile. CoRR, abs/1807.11626, 2018.

24. Esteban Real, Alok Aggarwal, Yanping Huang, and Quoc V. Le. Regularized evolution for image classifier architecture search. CoRR, abs/1802.01548, 2018.

25. Hanxiao Liu, Karen Simonyan, and Yiming Yang. DARTS: differentiable architecture search. CoRR, abs/1806.09055, 2018.

26. C. Szegedy, V. Vanhoucke, S. Ioffe, J. Shlens, and Z. Wojna. Rethinking the inception architecture for computer vision. In 2016 IEEE Conference on Computer Vision and Pattern Recognition (CVPR), pages 2818-2826, 2016.

27. F. Chollet. Xception: Deep learning with depthwise separable convolutions. In 2017 IEEE Conference on Computer Vision and Pattern Recognition (CVPR), pages 1800-1807, 2017 . 
28. Weiwen Jiang, Xinyi Zhang, Edwin Hsing-Mean Sha, Lei Yang, Qingfeng Zhuge, Yiyu Shi, and Jingtong Hu. Accuracy vs. efficiency: Achieving both through fpga-implementation aware neural architecture search. CoRR, abs/1901.11211, 2019.

29. Chen Zhang, Peng Li, Guangyu Sun, Yijin Guan, B. Xiao, and J. Cong. Optimizing fpga-based accelerator design for deep convolutional neural networks. In FPGA '15, 2015.

30. Vincent Vanhoucke, Andrew Senior, and Mark Z. Mao. Improving the speed of neural networks on cpus. In Deep Learning and Unsupervised Feature Learning Workshop, NIPS 2011, 2011.

31. Suyog Gupta, Ankur Agrawal, Kailash Gopalakrishnan, and Pritish Narayanan. Deep learning with limited numerical precision. volume 37 of Proceedings of Machine Learning Research, pages 1737-1746, Lille, France, 07-09 Jul 2015. PMLR.

32. Kevin Kiningham. Design and analysis of a hardware cnn accelerator. 2017.

33. Xuechao Wei, C. H. Yu, P. Zhang, Youxiang Chen, Y. Wang, H. Hu, Y. Liang, and J. Cong. Automated systolic array architecture synthesis for high throughput cnn inference on fpgas. 2017 54th ACM/EDAC/IEEE Design Automation Conference $(D A C)$, pages $1-6,2017$.

34. Yuhao Zhu, Matthew Mattina, and P. Whatmough. Mobile machine learning hardware at arm: A systems-on-chip (soc) perspective. ArXiv, abs/1801.06274, 2018.

35. Nallatech. FPGA Acceleration of Convolutional Neural Networks. https://www.nallatech.com/wp-content/uploads/Nalllatech-Whitepaper-FPGA-Accelerated-CNN-003TR.pdf.

36. Naveen Suda, Vikas Chandra, G. Dasika, Abinash Mohanty, Yu-Fei Ma, S. Vrudhula, Jae sun Seo, and Y. Cao. Throughput-optimized opencl-based fpga accelerator for large-scale convolutional neural networks. In FPGA '16, 2016.

37. Y. Wang, J. Xu, Y. Han, H. Li, and X. Li. Deepburning: Automatic generation of fpga-based learning accelerators for the neural network family. 2016 53nd ACM/EDAC/IEEE Design Automation Conference (DAC), pages 1-6, 2016.

38. Yann Le Cun, John S. Denker, and Sara A. Solla. Optimal brain damage. In Advances in Neural Information Processing Systems, pages 598-605. Morgan Kaufmann, 1990.

39. Babak Hassibi, David G. Stork, and Stork Crc. Ricoh. Com. Second order derivatives for network pruning: Optimal brain surgeon. In Advances in Neural Information Processing Systems 5, pages 164-171. Morgan Kaufmann, 1993.

40. Page A, Jafari A, Shea C, and Mohsenin T. Sparcnet: a hardware accelerator for efficient deployment of sparse convolutional networks. ACM Journal on Emerging Technologies in Computing Systems, 13(3)(31), 2017.

41. P. Molchanov, S. Tyree, Tero Karras, Timo Aila, and J. Kautz. Pruning convolutional neural networks for resource efficient inference. International Conference on Learning Representations (ICLR), 2017.

42. Song Han, Jeff Pool, John Tran, and William J. Dally. Learning both weights and connections for efficient neural networks. CoRR, abs/1506.02626, 2015.

43. Lance Elliot. Deep Compression and Pruning for Machine Learning in AI Self-Driving Cars: Using Convolutional Neural Networks (CNN). https://aitrends.com/ai-insider/deep-compressionpruning-machine-learning-ai-self-driving-cars-using- convolutional-neural-networks-cnn/, 2017.

44. Stephen Jose Hanson and Lorien Y. Pratt. Comparing biases for minimal network construction with back-propagation. In D. S. Touretzky, editor, Advances in Neural Information Processing Systems 1, pages 177-185. Morgan-Kaufmann, 1989.

45. Jiquan Ngiam, Zhenghao Chen, Daniel Chia, Pang W. Koh, Quoc V. Le, and Andrew Y. Ng. Tiled convolutional neural networks. In J. D. Lafferty, C. K. I. Williams, J. Shawe-Taylor, R. S. Zemel, and A. Culotta, editors, Advances in Neural Information Processing Systems 23, pages 1279-1287. Curran Associates, Inc., 2010.

46. B. Reagen, P. Whatmough, R. Adolf, S. Rama, H. Lee, S. K. Lee, J. M. Hernández-Lobato, G. Wei, and D. Brooks. Minerva: Enabling low-power, highly-accurate deep neural network accelerators. In 2016 ACM/IEEE 43rd Annual International Symposium on Computer Architecture (ISCA), pages 267-278, 2016.

47. Song Han, Huizi Mao, and William J. Dally. Deep Compression: Compressing Deep Neural Networks with Pruning, Trained Quantization and Huffman Coding. arXiv e-prints, page arXiv:1510.00149, October 2015.

48. Wenlin Chen, James T. Wilson, Stephen Tyree, Kilian Q. Weinberger, and Yixin Chen. Compressing neural networks with the hashing trick. CoRR, abs/1504.04788, 2015. 
49. Yunchao Gong, Liu Liu, Ming Yang, and Lubomir D. Bourdev. Compressing deep convolutional networks using vector quantization. CoRR, abs/1412.6115, 2014.

50. Emily L Denton, Wojciech Zaremba, Joan Bruna, Yann LeCun, and Rob Fergus. Exploiting linear structure within convolutional networks for efficient evaluation. In Z. Ghahramani, M. Welling, C. Cortes, N. D. Lawrence, and K. Q. Weinberger, editors, Advances in Neural Information Processing Systems 27, pages 1269-1277. Curran Associates, Inc., 2014.

51. X. Zhang, Jianhua Zou, Xiang Ming, K. He, and J. Sun. Efficient and accurate approximations of nonlinear convolutional networks. In 2015 IEEE Conference on Computer Vision and Pattern Recognition (CVPR), pages 1984-1992, 2015.

52. Max Jaderberg, Andrea Vedaldi, and Andrew Zisserman. Speeding up convolutional neural networks with low rank expansions. In Proceedings of the British Machine Vision Conference. BMVA Press, 2014.

53. Matthew D. Zeiler and R. Fergus. Visualizing and understanding convolutional networks. In European Conference on Computer Vision (ECCV), 2014.

54. Christian Szegedy, S. Ioffe, V. Vanhoucke, and Alexander Amir Alemi. Inception-v4, inception-resnet and the impact of residual connections on learning. In $A A A I, 2017$.

55. B. Wu, A. Wan, F. Iandola, P. H. Jin, and K. Keutzer. Squeezedet: Unified, small, low power fully convolutional neural networks for real-time object detection for autonomous driving. In 2017 IEEE Conference on Computer Vision and Pattern Recognition Workshops (CVPRW), pages 446-454, 2017.

56. Liang-Chieh Chen, George Papandreou, Florian Schroff, and Hartwig Adam. Rethinking atrous convolution for semantic image segmentation. CoRR, abs/1706.05587, 2017.

57. Gao Huang, Shichen Liu, L. V. D. Maaten, and Kilian Q. Weinberger. Condensenet: An efficient densenet using learned group convolutions. 2018 IEEE/CVF Conference on Computer Vision and Pattern Recognition, pages 2752-2761, 2018.

58. Jost Tobias Springenberg, A. Dosovitskiy, T. Brox, and Martin A. Riedmiller. Striving for simplicity: The all convolutional net. CoRR, abs/1412.6806, 2015.

59. A. Krizhevsky, , and G. Hinton. Learning Multiple Layers of Features from Tiny Images. Technical report, University of Toronto, 2009.

60. Yuval Netzer, Tao Wang, Adam Coates, Alessandro Bissacco, Bo Wu, and Andrew Y. Ng. Reading digits in natural images with unsupervised feature learning. In NIPS Workshop on Deep Learning and Unsupervised Feature Learning 2011, 2011.

61. Matthieu Courbariaux and Yoshua Bengio. Binarynet: Training deep neural networks with weights and activations constrained to +1 or -1 . ArXiv, abs/1602.02830, 2016.

62. Liangzhen Lai, Naveen Suda, and V. Chandra. Cmsis-nn: Efficient neural network kernels for arm cortex-m cpus. ArXiv, abs/1801.06601, 2018.

63. Liangzhen Lai, Naveen Suda, and V. Chandra. Deep convolutional neural network inference with floating-point weights and fixed-point activations. ArXiv, abs/1703.03073, 2017.

64. Jiantao Qiu, J. Wang, Song Yao, K. Guo, Boxun Li, Erjin Zhou, J. Yu, T. Tang, N. Xu, S. Song, Yu Wang, and H. Yang. Going deeper with embedded fpga platform for convolutional neural network. In FPGA '16, 2016.

65. Matthieu Courbariaux, Yoshua Bengio, and J. David. Training deep neural networks with low precision multiplications. arXiv:1412.7024, 2014.

66. Philipp Gysel. Ristretto: Hardware-oriented approximation of convolutional neural networks. ArXiv, abs/1605.06402, 2016.

67. H. Sharma, Jongse Park, Naveen Suda, Liangzhen Lai, Benson Chau, J. K. Kim, Vikas Chandra, and H. Esmaeilzadeh. Bit fusion: Bit-level dynamically composable architecture for accelerating deep neural network. 2018 ACM/IEEE 45th Annual International Symposium on Computer Architecture (ISCA), pages 764-775, 2018.

68. Subarna Tripathi, Gökçe Dane, B. Kang, V. Bhaskaran, and T. Nguyen. Lcdet: Low-complexity fully-convolutional neural networks for object detection in embedded systems. 2017 IEEE Conference on Computer Vision and Pattern Recognition Workshops (CVPRW), pages 411-420, 2017.

69. Matthieu Courbariaux, Yoshua Bengio, and Jean-Pierre David. Binaryconnect: Training deep neural networks with binary weights during propagations. In C. Cortes, N. Lawrence, D. Lee, M. Sugiyama, and R. Garnett, editors, Advances in Neural Information Processing Systems, volume 28, pages 3123-3131. Curran Associates, Inc., 2015.

70. Yixing Li, Zichuan Liu, Kai Xu, Hao Yu, and Fengbo Ren. A gpu-outperforming fpga accelerator architecture for binary convolutional neural networks. ACM Journal on Emerging Technologies in Computing Systems, 14(2), July 2018. 
71. Bradley McDanel, Surat Teerapittayanon, and H.T. Kung. Embedded binarized neural networks. In Proceedings of the 2017 International Conference on Embedded Wireless Systems and Networks, EWSN '17, page 168-173, USA, 2017. Junction Publishing.

72. Ruizhou Ding, Z. Liu, Rongye Shi, Diana Marculescu, and R. Blanton. Lightnn: Filling the gap between conventional deep neural networks and binarized networks. Proceedings of the on Great Lakes Symposium on VLSI 2017, 2017.

73. Mohammad Rastegari, Vicente Ordonez, Joseph Redmon, and Ali Farhadi. Xnor-net: Imagenet classification using binary convolutional neural networks. In Bastian Leibe, Jiri Matas, Nicu Sebe, and Max Welling, editors, Computer Vision - ECCV 2016, pages 525-542, Cham, 2016. Springer International Publishing.

74. Shuchang Zhou, Zekun Ni, X. Zhou, He Wen, Yuxin Wu, and Yuheng Zou. Dorefa-net: Training low bitwidth convolutional neural networks with low bitwidth gradients. ArXiv, abs/1606.06160, 2016

75. Itay Hubara, Matthieu Courbariaux, Daniel Soudry, Ran El-Yaniv, and Yoshua Bengio. Quantized neural networks: Training neural networks with low precision weights and activations. J. Mach. Learn. Res., 18:187:1-187:30, 2017.

76. J. Wu, C. Leng, Yuhang Wang, Q. Hu, and J. Cheng. Quantized convolutional neural networks for mobile devices. 2016 IEEE Conference on Computer Vision and Pattern Recognition (CVPR), pages 4820-4828, 2016.

77. Geoffrey E. Hinton, Oriol Vinyals, and J. Dean. Distilling the knowledge in a neural network. ArXiv, abs/1503.02531, 2015.

78. A. Romero, Nicolas Ballas, S. Kahou, Antoine Chassang, C. Gatta, and Yoshua Bengio. Fitnets: Hints for thin deep nets. CoRR, abs/1412.6550, 2015.

79. Liang $\mathrm{Lu}$, Michelle Guo, and S. Renals. Knowledge distillation for small-footprint highway networks. 2017 IEEE International Conference on Acoustics, Speech and Signal Processing (ICASSP), pages 4820-4824, 2017.

80. R. Srivastava, Klaus Greff, and J. Schmidhuber. Highway networks. ArXiv, abs/1505.00387, 2015.

81. A. Mishra and Debbie Marr. Apprentice: Using knowledge distillation techniques to improve low-precision network accuracy. ArXiv, abs/1711.05852, 2018.

82. Vijay Badrinarayanan, Alex Kendall, and R. Cipolla. Segnet: A deep convolutional encoder-decoder architecture for image segmentation. IEEE Transactions on Pattern Analysis and Machine Intelligence, 39:2481-2495, 2017.

83. Jie Hu, L. Shen, Samuel Albanie, Gang Sun, and Enhua Wu. Squeeze-and-excitation networks. IEEE Transactions on Pattern Analysis and Machine Intelligence, 42:2011-2023, 2020.

84. Saining Xie, Ross B. Girshick, Piotr Dollár, Zhuowen Tu, and Kaiming He. Aggregated residual transformations for deep neural networks. 2017 IEEE Conference on Computer Vision and Pattern Recognition (CVPR), pages 5987-5995, 2017.

85. Hieu Pham, Melody Guan, Barret Zoph, Quoc Le, and Jeff Dean. Efficient neural architecture search via parameters sharing. volume 80 of Proceedings of Machine Learning Research, pages 4095-4104, Stockholmsmässan, Stockholm Sweden, 10-15 Jul 2018. PMLR.

86. Yanchen Wang. Deep learning in real time - inference acceleration and continuous training. https://medium.com/syncedreview/deep-learning-inreal-time-inference-acceleration-and-continuous- training-17dac9438b0b, 2017.

87. Tianshi Chen, Zidong Du, Ninghui Sun, J. Wang, Chengyong Wu, Yunji Chen, and Olivier Temam. Diannao: a small-footprint high-throughput accelerator for ubiquitous machine-learning. In $A S P L O S$ '14, 2014.

88. Yunji Chen, T. Luo, Shaoli Liu, S. Zhang, Liqiang He, J. Wang, L. Li, Tianshi Chen, Z. Xu, Ninghui Sun, and O. Temam. Dadiannao: A machine-learning supercomputer. 2014 47th Annual IEEE/ACM International Symposium on Microarchitecture, pages 609-622, 2014.

89. Yu-Hsin Chen, T. Krishna, J. Emer, and V. Sze. Eyeriss: An energy-efficient reconfigurable accelerator for deep convolutional neural networks. IEEE Journal of Solid-State Circuits, 52:127-138, 2017.

90. Y. Chen, J. Emer, and V. Sze. Eyeriss v2: A flexible and high-performance accelerator for emerging deep neural networks. ArXiv, abs/1807.07928, 2018.

91. Hyunsun Park, Dongyoung Kim, Junwhan Ahn, and Sungjoo Yoo. Zero and data reuse-aware fast convolution for deep neural networks on gpu. 2016 International Conference on Hardware/Software Codesign and System Synthesis (CODES+ISSS), pages 1-10, 2016. 
92. Song Han, Xingyu Liu, Huizi Mao, J. Pu, A. Pedram, M. Horowitz, and W. Dally. Eie: Efficient inference engine on compressed deep neural network. 2016 ACM/IEEE 43rd Annual International Symposium on Computer Architecture (ISCA), pages 243-254, 2016.

93. Dawei Li, Xiaolong Wang, and Deguang Kong. Deeprebirth: Accelerating deep neural network execution on mobile devices. ArXiv, abs/1708.04728, 2018.

94. A. Aimar, Hesham Mostafa, Enrico Calabrese, Antonio Rios-Navarro, Ricardo Tapiador-Morales, I. Lungu, Moritz B. Milde, F. Corradi, A. Linares-Barranco, Shih-Chii Liu, and T. Delbruck. Nullhop: A flexible convolutional neural network accelerator based on sparse representations of feature maps. IEEE Transactions on Neural Networks and Learning Systems, 30:644-656, 2019.

95. Kalin Ovtcharov, Olatunji Ruwase, J. Kim, Jeremy Fowers, K. Strauss, and E. Chung. Accelerating deep convolutional neural networks using specialized hardware. 2015.

96. Maurice Peemen, Arnaud A. A. Setio, B. Mesman, and H. Corporaal. Memory-centric accelerator design for convolutional neural networks. 2013 IEEE 31st International Conference on Computer Design (ICCD), pages 13-19, 2013.

97. N. Jouppi et al. In-datacenter performance analysis of a tensor processing unit. 2017 ACM/IEEE 44th Annual International Symposium on Computer Architecture (ISCA), pages $1-12,2017$.

98. Y. Shen, Michael Ferdman, and P. Milder. Maximizing cnn accelerator efficiency through resource partitioning. 2017 ACM/IEEE 44th Annual International Symposium on Computer Architecture (ISCA), pages 535-547, 2017.

99. U. Aydonat, S. O'Connell, D. Capalija, A. Ling, and Gordon R. Chiu. An opencl ${ }^{\mathrm{TM}}$ deep learning accelerator on arria 10. In FPGA '17, 2017.

100. L. Lu, Y. Liang, Qingcheng Xiao, and Shengen Yan. Evaluating fast algorithms for convolutional neural networks on fpgas. 2017 IEEE 25th Annual International Symposium on Field-Programmable Custom Computing Machines (FCCM), pages 101-108, 2017.

101. Jialiang Zhang and J. Li. Improving the performance of opencl-based fpga accelerator for convolutional neural network. In FPGA '17, 2017.

102. Ruizhe Zhao, Xinyu Niu, Yajie Wu, W. Luk, and Q. Liu. Optimizing cnn-based object detection algorithms on embedded fpga platforms. In $A R C, 2017$.

103. Arish S., Sharad Sinha, and Smitha K.G. Optimization of Convolutional Neural Networks on Resource Constrained Devices. In 2019 IEEE Computer Society Annual Symposium on VLSI (ISVLSI), pages 19-24, 2019.

104. J. Albericio, A. Delmas, P. Judd, Sayeh Sharify, G. O'Leary, R. Genov, and A. Moshovos. Bit-pragmatic deep neural network computing. 2017 50th Annual IEEE/ACM International Symposium on Microarchitecture (MICRO), pages 382-394, 2017.

105. Angshuman Parashar, Minsoo Rhu, Anurag Mukkara, Antonio Puglielli, R. Venkatesan, B. Khailany, J. Emer, Stephen W. Keckler, and W. Dally. Scnn: An accelerator for compressed-sparse convolutional neural networks. 2017 ACM/IEEE 44th Annual International Symposium on Computer Architecture (ISCA), pages 27-40, 2017.

106. Stylianos I. Venieris and C. Bouganis. fpgaconvnet: A framework for mapping convolutional neural networks on fpgas. 2016 IEEE 24th Annual International Symposium on Field-Programmable Custom Computing Machines (FCCM), pages 40-47, 2016.

107. Mohammad Motamedi, Philipp Gysel, V. Akella, and S. Ghiasi. Design space exploration of fpga-based deep convolutional neural networks. 2016 21st Asia and South Pacific Design Automation Conference (ASP-DAC), pages 575-580, 2016.

108. Mohammad Motamedi, Philipp Gysel, and S. Ghiasi. Placid: A platform for fpga-based accelerator creation for dcnns. ACM Transactions on Multimedia Computing, Communications, and Applications (TOMM), 13:1 - 21, 2017.

109. Dimitrios Stamoulis, Ruizhou Ding, Di Wang, Dimitrios Lymberopoulos, Bodhi Priyantha, Jie Liu, and Diana Marculescu. Single-path nas: Designing hardware-efficient convnets in less than 4 hours. In Ulf Brefeld, Elisa Fromont, Andreas Hotho, Arno Knobbe, Marloes Maathuis, and Céline Robardet, editors, Machine Learning and Knowledge Discovery in Databases, pages 481-497, Cham, 2020. Springer International Publishing.

110. Bichen Wu, Xiaoliang Dai, Peizhao Zhang, Yanghan Wang, Fei Sun, Yiming Wu, Yuandong Tian, Peter Vajda, Yangqing Jia, and Kurt Keutzer. Fbnet: Hardware-aware efficient convnet design via differentiable neural architecture search. CoRR, abs/1812.03443, 2018. 
111. Han Cai, Ligeng Zhu, and Song Han. Proxylessnas: Direct neural architecture search on target task and hardware. CoRR, abs/1812.00332, 2018.

112. Qing $\mathrm{Lu}$, Weiwen Jiang, Xiaowei Xu, Yiyu Shi, and Jingtong Hu. On neural architecture search for resource-constrained hardware platforms, 2019.

113. Weiwen Jiang, Lei Yang, Sakyasingha Dasgupta, Jingtong Hu, and Yiyu Shi. Standing on the shoulders of giants: Hardware and neural architecture co-search with hot start, 2020 .

114. Yuhong Li, Cong Hao, Xiaofan Zhang, Xinheng Liu, Yao Chen, Jinjun Xiong, Wen mei Hwu, and Deming Chen. Edd: Efficient differentiable dnn architecture and implementation co-search for embedded ai solutions, 2020.

115. X. Zhang, J. Wang, C. Zhu, Y. Lin, J. Xiong, W. Hwu, and D. Chen. Dnnbuilder: an automated tool for building high-performance dnn hardware accelerators for fpgas. In 2018 IEEE/ACM International Conference on Computer-Aided Design (ICCAD), pages $1-8,2018$.

116. Cong Hao, Yao Chen, Xinheng Liu, Atif Sarwari, Daryl Sew, Ashutosh Dhar, Bryan Wu, Dongdong Fu, Jinjun Xiong, Wen mei Hwu, Junli Gu, and Deming Chen. Nais: Neural architecture and implementation search and its applications in autonomous driving, 2019.

117. Alberto Marchisio, Andrea Massa, Vojtech Mrazek, Beatrice Bussolino, Maurizio Martina, and Muhammad Shafique. Nascaps: A framework for neural architecture search to optimize the accuracy and hardware efficiency of convolutional capsule networks, 2020.

118. P. Achararit, M. A. Hanif, R. V. W. Putra, M. Shafique, and Y. Hara-Azumi. Apnas: Accuracy-and-performance-aware neural architecture search for neural hardware accelerators. IEEE Access, 8:165319-165334, 2020.

119. Weiwen Jiang, Lei Yang, Edwin Hsing-Mean Sha, Qingfeng Zhuge, Shouzhen Gu, Yiyu Shi, and Jingtong Hu. Hardware/software co-exploration of neural architectures. CoRR, abs/1907.04650, 2019.

120. Cong Hao, Xiaofan Zhang, Yuhong Li, Sitao Huang, Jinjun Xiong, Kyle Rupnow, Wen-Mei Hwu, and Deming Chen. FPGA/DNN co-design: An efficient design methodology for iot intelligence on the edge. CoRR, abs/1904.04421, 2019.

121. Xiaofan Zhang, Haoming Lu, Cong Hao, Jiachen Li, Bowen Cheng, Yuhong Li, Kyle Rupnow, Jinjun Xiong, Thomas Huang, Honghui Shi, Wen mei Hwu, and Deming Chen. Skynet: a hardware-efficient method for object detection and tracking on embedded systems, 2020.

122. M. Abadi et al. Tensorflow: Large-scale machine learning on heterogeneous distributed systems. ArXiv, abs/1603.04467, 2016.

123. François Chollet et al. Keras: The Python Deep Learning library. https://keras.io, 2018.

124. Adam Paszke, S. Gross, Soumith Chintala, G. Chanan, E. Yang, Zachary Devito, Zeming Lin, Alban Desmaison, L. Antiga, and A. Lerer. Automatic differentiation in PyTorch. In NIPS Workshop, 2017.

125. Yangqing Jia, Evan Shelhamer, Jeff Donahue, Sergey Karayev, Jonathan Long, Ross Girshick, Sergio Guadarrama, and Trevor Darrell. Caffe: Convolutional architecture for fast feature embedding. In Proceedings of the 22nd ACM International Conference on Multimedia, MM '14, page 675-678, New York, NY, USA, 2014. Association for Computing Machinery.

126. Junli Gu, Y. Liu, Yuan Gao, and Maohua Zhu. Opencl caffe: Accelerating and enabling a cross platform machine learning framework. In Proceedings of the 4 th International Workshop on OpenCL, 2016.

127. Shasha Guo, L. Wang, B. Chen, Qiang Dou, Yuxing Tang, and Zhisheng Li. Fixcaffe: Training cnn with low precision arithmetic operations by fixed point caffe. In APPT, 2017.

128. Rami Al-Rfou et al. Theano: A python framework for fast computation of mathematical expressions. ArXiv, abs/1605.02688, 2016.

129. Travis E and Oliphant. A guide to NumPy. USA: Trelgol Publishing, 2006.

130. nVIDIA. Tensorrt userguide. nVidia Corporation, 2017.

131. Robert David, Jared Duke, Advait Jain, Vijay Janapa Reddi, Nat Jeffries, Jian Li, Nick Kreeger, Ian Nappier, Meghna Natraj, Shlomi Regev, Rocky Rhodes, Tiezhen Wang, and Pete Warden. Tensorflow lite micro: Embedded machine learning on tinyml systems, 2020.

132. Ji Lin, Wei-Ming Chen, Yujun Lin, John Cohn, Chuang Gan, and Song Han. Mcunet: Tiny deep learning on iot devices, 2020.

133. Microsoft. Embedded Learning Library, 2020. URL https://microsoft.github.io/ELL/. 
134. uTensor. uTensor, 2020. URL https://github.com/uTensor/uTensor.

135. Nadav Rotem, Jordan Fix, Saleem Abdulrasool, Garret Catron, Summer Deng, Roman Dzhabarov, Nick Gibson, James Hegeman, Meghan Lele, Roman Levenstein, Jack Montgomery, Bert Maher, Satish Nadathur, Jakob Olesen, Jongsoo Park, Artem Rakhov, Misha Smelyanskiy, and Man Wang. Glow: Graph lowering compiler techniques for neural networks, 2019.

136. STMicroelectronics. STM32Cube.AI, 2020. URL https://www.st.com/content/st com/en/stm32-ann.html.

137. T. Chen, Mu Li, Y. Li, M. Lin, Naiyan Wang, Minjie Wang, Tianjun Xiao, B. Xu, C. Zhang, and Zheng Zhang. Mxnet: A flexible and efficient machine learning library for heterogeneous distributed systems. ArXiv, abs/1512.01274, 2015.

138. C. Zhang, Zhenman Fang, Peipei Zhou, P. Pan, and J. Cong. Caffeine: Towards uniformed representation and acceleration for deep convolutional neural networks. 2016 IEEE/ACM International Conference on Computer-Aided Design (ICCAD), pages 1-8, 2016.

139. Clive Maxfield. Push-button generation of deep neural networks. https://www.embedded.com/push-button-generation-of-deep-neural-networks/, 2016.

140. Yaman Umuroglu, Nicholas J. Fraser, G. Gambardella, M. Blott, P. Leong, Magnus Jahre, and K. Vissers. Finn: A framework for fast, scalable binarized neural network inference. In FPGA '17, 2017.

141. K. Guo, L. Sui, J. Qiu, J. Yu, J. Wang, S. Yao, S. Han, Y. Wang, and H. Yang. Angel-eye: A complete design flow for mapping cnn onto embedded fpga. IEEE Transactions on Computer-Aided Design of Integrated Circuits and Systems, 37(1):35-47, 2018.

142. Alexandros Kouris, Stylianos I. Venieris, and C. Bouganis. Cascadecnn: Pushing the performance limits of quantisation in convolutional neural networks. 2018 28th International Conference on Field Programmable Logic and Applications (FPL), pages 155-1557, 2018.

143. Junzhong Shen, Y. Huang, Zelong Wang, Yuran Qiao, Mei Wen, and C. Zhang. Towards a uniform template-based architecture for accelerating $2 \mathrm{~d}$ and $3 \mathrm{~d}$ cnns on fpga. Proceedings of the 2018 ACM/SIGDA International Symposium on Field-Programmable Gate Arrays, 2018.

144. Y. Lai, Yuze Chi, Yuwei Hu, J. Wang, C. H. Yu, Yuan Zhou, J. Cong, and Z. Zhang. Heterocl: A multi-paradigm programming infrastructure for software-defined reconfigurable computing. Proceedings of the 2019 ACM/SIGDA International Symposium on Field-Programmable Gate Arrays, 2019.

145. J. Xu, Zhiqiang Liu, Jingfei Jiang, Y. Dou, and S. Li. Cafpga: An automatic generation model for cnn accelerator. Microprocess. Microsystems, 60:196-206, 2018.

146. H. Sharma, J. Park, D. Mahajan, E. Amaro, J. K. Kim, C. Shao, A. Mishra, and H. Esmaeilzadeh. From high-level deep neural models to fpgas. In 201649 th Annual IEEE/ACM International Symposium on Microarchitecture (MICRO), pages 1-12, 2016.

147. Pengfei Xu, Xiaofan Zhang, Cong Hao, Yang Zhao, Yongan Zhang, Yue Wang, Chaojian Li, Zetong Guan, Deming Chen, and Yingyan Lin. Autodnnchip: An automated dnn chip predictor and builder for both fpgas and asics. The 2020 ACM/SIGDA International Symposium on Field-Programmable Gate Arrays, Feb 2020.

148. H. Zeng, Ren Chen, C. Zhang, and V. Prasanna. A framework for generating high throughput cnn implementations on fpgas. Proceedings of the 2018 ACM/SIGDA International Symposium on Field-Programmable Gate Arrays, 2018.

149. Y. Ma, Naveen Suda, Yu Cao, Jae sun Seo, and S. Vrudhula. Scalable and modularized rtl compilation of convolutional neural networks onto fpga. 2016 26th International Conference on Field Programmable Logic and Applications (FPL), pages 1-8, 2016.

150. Y. Ma, Y. Cao, S. Vrudhula, and J. Seo. An automatic rtl compiler for high-throughput fpga implementation of diverse deep convolutional neural networks. In 2017 27th International Conference on Field Programmable Logic and Applications (FPL), pages $1-8,2017$.

151. Y. Guan, H. Liang, N. Xu, W. Wang, S. Shi, X. Chen, G. Sun, W. Zhang, and J. Cong. Fp-dnn: An automated framework for mapping deep neural networks onto fpgas with rtl-hls hybrid templates. In 2017 IEEE 25th Annual International Symposium on Field-Programmable Custom Computing Machines (FCCM), pages 152-159, 2017.

152. Yufei Ma, Naveen Suda, Yu Cao, Sarma Vrudhula, and Jae sun Seo. Alamo: Fpga acceleration of deep learning algorithms with a modularized rtl compiler. Integration, $62: 14-23,2018$. 
153. V. Gokhale, A. Zaidy, A. X. M. Chang, and E. Culurciello. Snowflake: An efficient hardware accelerator for convolutional neural networks. In 2017 IEEE International Symposium on Circuits and Systems (ISCAS), pages 1-4, 2017.

154. Radosław Cieszewski, Maciej Linczuk, Krzysztof Pozniak, and Ryszard Romaniuk. Review of parallel computing methods and tools for FPGA technology. In Ryszard S. Romaniuk, editor, Photonics Applications in Astronomy, Communications, Industry, and High-Energy Physics Experiments 2013, volume 8903, pages 596 - 608. International Society for Optics and Photonics, SPIE, 2013.

155. Bo Joel Svensson and Rakesh Tripathi. Getting started with opencl on the zynq, 2016.

156. F. Muslim, L. Ma, Medhi Roozmeh, and L. Lavagno. Efficient fpga implementation of opencl high-performance computing applications via high-level synthesis. IEEE Access, 5:2747-2762, 2017.

157. R. Cieszewski, K. Pozniak, and Ryszard S. Romaniuk. Python based high-level synthesis compiler. In Proc. SPIE 9290, Photonics Applications in Astronomy, Communications, Industry, and High-Energy Physics Experiments, 2014.

158. Radosław Cieszewski and Maciej Linczuk. Rpython high-level synthesis. In Symposium on Photonics Applications in Astronomy, Communications, Industry, and High-Energy Physics Experiments (WILGA), 2016.

159. Jan Decaluwe. Myhdl: a python-based hardware description language. Linux Journal, 2004:5, 2004

160. D. Lockhart, Gary Zibrat, and Christopher Batten. Pymtl: A unified framework for vertically integrated computer architecture research. 2014 47th Annual IEEE/ACM International Symposium on Microarchitecture, pages 280-292, 2014.

161. Per Haglund, O. Mencer, W. Luk, and Benjamin Tai. Hardware design with a scripting language. In FPL, 2003.

162. John Clow, Georgios Tzimpragos, D. Dangwal, Sammy Guo, Joseph McMahan, and T. Sherwood. A pythonic approach for rapid hardware prototyping and instrumentation. 2017 27th International Conference on Field Programmable Logic and Applications (FPL), pages $1-7,2017$.

163. Ali José Mashtizadeh. Phdl : a python hardware design framework. 2007.

164. S. Merchant, G. D. Peterson, Sang Ki Park, and S. G. Kong. Fpga implementation of evolvable block-based neural networks. In 2006 IEEE International Conference on Evolutionary Computation, pages 3129-3136, 2006. 